ARTICLE

\title{
Rab1-AMPylation by Legionella DrrA is allosterically activated by Rab1
}

\author{
Jiqing Du (10) 1,2,8, Marie-Kristin von Wrisberg (10) 3,8, Burak Gulen (10) 1,2, Matthias Stahl (10 1,7, Christian Pett (1) 4 , \\ Christian Hedberg ${ }^{4}$, Kathrin Lang (i) ${ }^{3 凶}$, Sabine Schneider (iD ${ }^{5 凶}$ \& Aymelt Itzen (i) ${ }^{1,2,6 凶}$
}

Legionella pneumophila infects eukaryotic cells by forming a replicative organelle - the Legionella containing vacuole. During this process, the bacterial protein DrrA/SidM is secreted and manipulates the activity and post-translational modification (PTM) states of the vesicular trafficking regulator Rab1. As a result, Rab1 is modified with an adenosine monophosphate (AMP), and this process is referred to as AMPylation. Here, we use a chemical approach to stabilise low-affinity Rab:DrrA complexes in a site-specific manner to gain insight into the molecular basis of the interaction between the Rab protein and the AMPylation domain of DrrA. The crystal structure of the Rab:DrrA complex reveals a previously unknown non-conventional Rab-binding site (NC-RBS). Biochemical characterisation demonstrates allosteric stimulation of the AMPylation activity of DrrA via Rab binding to the NC-RBS. We speculate that allosteric control of DrrA could in principle prevent random and potentially cytotoxic AMPylation in the host, thereby perhaps ensuring efficient infection by Legionella.

\footnotetext{
${ }^{1}$ Center for Integrated Protein Science Munich (CIPSM), Department of Chemistry, Technical University of Munich, Garching 85748, Germany. ${ }^{2}$ Center for Experimental Medicine, Institute of Biochemistry and Signal Transduction, Universitätsklinikum Hamburg-Eppendorf (UKE), Hamburg 20246, Germany. ${ }^{3}$ Center for Integrated Protein Science Munich (CIPSM), Department of Chemistry, Technical University of Munich, Institute for Advanced Study, Garching 85748, Germany. ${ }^{4}$ Chemical Biology Center (KBC), Department of Chemistry, Umeå University, Linnaeus väg 10, 90187 Umeå, Sweden. ${ }^{5}$ Center for Integrated Protein Science Munich (CIPSM), Department of Chemistry, Ludwig-Maximilians-University Munich, München 81377, Germany. ${ }^{6}$ Center for Structural Systems Biology (CSSB), University Medical Centre Hamburg-Eppendorf (UKE), Hamburg, Germany. ${ }^{7}$ Present address: Science for Life Laboratory, Department of Oncology-Pathology, Karolinska Institutet, Box 1031, 17121 Solna, Stockholm, Sweden. ${ }^{8}$ These authors contributed equally: Jiqing Du, MarieKristin von Wrisberg. ${ }^{\circledR}$ email: kathrin.lang@tum.de; sabine.schneider@cup.Imu.de; a.itzen@uke.de
} 
T he Gram-negative bacterium Legionella pneumophila is the causative agent of Legionnaires' disease. After uptake by human alveolar macrophages via phagocytosis, the pathogen establishes a replicative organelle referred to as the Legionella-containing vacuole $(\mathrm{LCV})^{1}$. The formation and maintenance of the LCV are mediated by $\sim 330$ Legionella effector proteins that are released by the bacterial Type IVb secretion system (T4bSS) from the bacterium into the host. These effectors interfere with different host processes, including manipulation of vesicular trafficking (summarised in ref. ${ }^{2}$ ). In particular, one intensely studied protein, Legionella effector DrrA (Defect in Rab1 recruitment A, also referred to as SidM, substrate of $\mathrm{Icm} /$ Dot), is secreted early during infection, and it manipulates the vesicular trafficking regulator Rab1 (Ras-related protein Rab-1).

Rab1 is a member of the Rab family of small GTPases that are involved in spatially and temporally regulating intracellular vesicular trafficking between organelles. Rab proteins act as molecular switches that exist in inactive guanosine diphosphate (GDP) and active guanosine triphosphate (GTP) bound states ${ }^{3}$. At the structural level, the activity state is communicated to interaction partners via two important regulatory loop regions referred to as switch I and switch II. These regions are conformationally flexible in the inactive state but they become structurally ordered in the active form ${ }^{4}$. Only in the active form, Rab proteins mediate signalling via the recruitment of GTP statespecific effector molecules. Guanine nucleotide exchange factors (GEFs) promote GTP loading of Rab, whereas GTPase activating proteins (GAPs) stimulate the intrinsic GTP hydrolysis activity and return Rab back to the inactive state ${ }^{3}$. Furthermore, Rab proteins are post-translationally modified with geranylgeranyl lipids at the structurally flexible C-terminus, enabling reversible membrane attachment ${ }^{5}$. The recycling of geranylgeranylated Rabs from the membrane occurs in the inactive state and is controlled by GDP dissociation inhibitors (GDIs). The biology of Rab proteins has been the topic of a recent review ${ }^{3}$.

Due to their pivotal role in regulating vesicular trafficking, Rab proteins are frequently targeted and manipulated by bacterial pathogens. Rab1 controls vesicular transport from the endoplasmic reticulum (ER) to the Golgi apparatus ${ }^{6,7}$. However, it is subjected to intense manipulation during Legionella infections and is rerouted from the ER to the LCV. In this process, Rab1 is known to be targeted by six different Legionella proteins, one of which is $\operatorname{DrrA}^{2,8,9}$. However, the deletion of DrrA has little impact on successful infection by Legionella, probably due to effector redundancy ${ }^{9}$.

DrrA consists of three functional domains (Fig. 1a). The Cterminal phosphatidylinositol-4-phosphate (PI4P) binding site (referred to as P4M) mediates binding to the PI4P-positive LCV membrane ${ }^{10,11}$. A central Rab1-GEF domain recruits Rab1 via GDP-to-GTP exchange $e^{8,9,12}$. The $\mathrm{N}$-terminal domain possesses AMPylation activity (also referred to as adenylylation) ${ }^{13}$. AMPylation is a post-translational modification (PTM) in which an adenosine triphosphate (ATP) is utilised in order to transfer adenosine monophosphate (AMP) to hydroxyl-bearing amino acid side chains. The N-terminal AMP-transferase (ATase) activity of DrrA preferentially mediates AMPylation of active Rab1b at $\mathrm{Y} 7_{\text {Rablb }}$ (i.e. $\mathrm{Y}_{\text {Rabla }}$ in Rabla), which is located at the C-terminal end of the important Rab1 switch II region ${ }^{13,14}$. AMPylation inhibits binding to interaction partners such as GDI and molecule interacting with CasL 3 (MICAL3). Also, GAP-catalysed GTP hydrolysis by Rab1 is impaired, thereby rendering the protein permanently bound to GTP when AMPylated ${ }^{13,15}$. Notably, it was reported that GEF-deficient DrrA mutants are competent in the recruitment of Rabl from Rab1:GDI complex to the $\mathrm{LCV}^{16}$.

The structure of DrrA has been partially characterised ${ }^{11-13,17}$. However, the structure of the full ATase domain and the mechanism of Rablb AMPylation remain elusive. The ATase domain belongs to the DNA polymerase $\beta$-like enzyme family and shares structural similarity with the enzyme glutamine synthetase adenylyl transferase (GS-ATase) from Escherichia coli ${ }^{18}$. In this class of enzymes, three acidic amino acid residues $\left(\mathrm{D} 11_{\mathrm{DrrA}}, \mathrm{D} 11_{\mathrm{DrrA}}\right.$ and D150 $\left.0_{\text {DrrA }}\right)$ are involved in coordinating an essential $\mathrm{Mg}^{2+}$ relevant for ATP binding and AMP transfer. The structural basis for AMP transfer and Rab1 binding has not yet been reported, since structural investigation of Rab1DrrA $_{\text {ATase }}$ complexes is hampered by inherently low affinity; the Michaelis constant $\left(K_{\mathrm{M}}\right)$ is only $\sim 64 \mu \mathrm{M}^{14}$.

Herein, we employ covalent crosslinking approaches to stabilise and characterise low-affinity DrrA $\mathrm{ATase}_{\mathrm{As}}$ :Rab-complexes. The crystal structure of a DrrA $A_{\text {ATase }}:$ Rab-complex reveals an unrecognised regulatory Rab binding site. By mutational analysis, mass spectrometry and activity determinations we show that binding of Rab to this particular position on DrrA acts as a safety switch to control the AMPylation activity of DrrA via an allosteric mechanism in vitro. Our findings lead us to speculate that a potentially harmful AMPylation activity of DrrA may be controlled by Rab1:GTP-binding to an allosteric site.

\section{Results}

Conceptual design for trapping the DrrA-Rab complex. Since the ATase domain of DrrA binds to Rablb only with low affinity $^{14}$, we attempted to covalently trap and thereby homogenously enrich and stabilise the complex via two different chemical approaches. We first employed a crosslinking strategy that we recently developed based on site-specific incorporation of unnatural amino acids (UAAs) bearing bromoalkyl moieties (e.g. BrC6K, Fig. 1b) that react specifically with nucleophilic natural amino acids (e.g. Cys, Asp and Glu) in a proximity-enhanced manner upon complex formation ${ }^{19-21}$. The main effect of proximity consists in increasing the effective local concentration of the reactants to boost reaction rates and enable reactions that would not yield products in the absence of the concentration effect. To stabilise the $\operatorname{DrrA}_{\mathrm{ATase}}-\mathrm{Rab} 1 \mathrm{~b}$ interface, we overexpressed various C-terminally His6-tagged Rab1b constructs (amino acids 3-174; Rab1b ${ }_{3-174}$ with a Q67 $\mathrm{A}_{\mathrm{Rab} 1 \mathrm{~b}}$ mutation that minimises Rab1b GTP hydrolysis) ${ }^{22,23}$ bearing BrC6K at different positions ( $\mathrm{R} 69_{\mathrm{Rab} 1 \mathrm{~b}}, \mathrm{~T} 72_{\mathrm{Rab} 1 \mathrm{~b}}, \mathrm{I} 73_{\mathrm{Rab} 1 \mathrm{~b}}$ and $\mathrm{R} 79_{\mathrm{Rab} 1 \mathrm{~b}}$ ) in the vicinity of the AMPylation site $\left(\mathrm{Y} 77_{\mathrm{Rab} 1 \mathrm{~b}}\right)$, together with an $\mathrm{N}$ terminally Strep-tagged wild type (wt) $\operatorname{DrrA}_{\mathrm{ATase}}$ construct (amino acids 16-352; $\operatorname{DrrA}_{16-352}$ ). Co-expression of Rablb with BrC6K at position $69_{\mathrm{Rab} 1 b}$ and wt $\operatorname{DrrA}_{\mathrm{AT} \text { Tase }}$ resulted in the formation of a covalently crosslinked complex, as confirmed by sodium dodecyl sulphate polyacrylamide gel electrophoresis (SDS-PAGE) shift analysis via $\alpha$-His6 and $\alpha$-Strep western blotting (Fig. 1c and Supplementary Fig. 1). We purified the crosslinked complex by affinity and size-exclusion chromatography, and performed tandem mass spectrometry (MS/MS), which indicated that the crosslink corresponds to an ester linkage formed between BrC6K at position $69_{\text {Rab1b }}$ within Rab1b and D82 2 DrrA within DrrA (Supplementary Fig. 2 and Supplementary Table 1). This finding was corroborated by mutagenesis experiments, in which the D82 $\mathrm{A}_{\mathrm{DrrA}}$ mutation completely abolished crosslink formation, while mutating the Asp residue with the more nucleophilic Cys led to quantitatively thioether crosslinked Rablb:DrrA complex (Fig. 1c and Supplementary Fig. 1). Electrospray ionisation MS (ESI-MS) of the purified complex formed in living E. coli cells between $\mathrm{R} 69 \mathrm{BrC} 6 \mathrm{~K}_{\mathrm{Rab} 1 \mathrm{~b}}$ and $\mathrm{D} 82 \mathrm{C}_{\text {Drra }}$ verified the identity of the thioether crosslinked complex, which is AMPylated at $Y 77_{\text {Rablb }}$ within Rablb, confirming the enzyme and substrate activity of both DrrA and Rablb mutants (Fig. 1d). Intriguingly, amino acid D82 $2_{\operatorname{Drr}}$ in DrrA is not positioned in the 

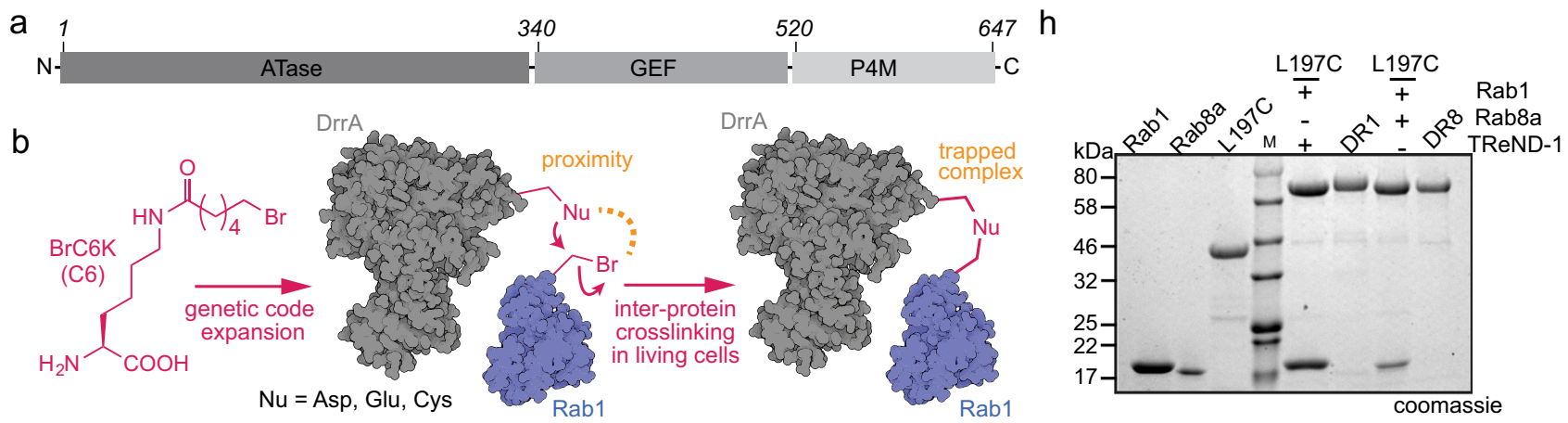

C

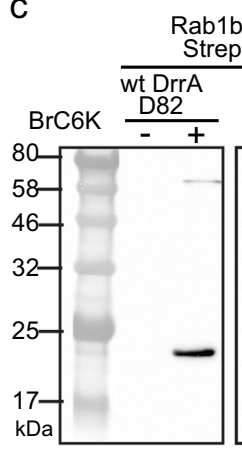

Rab1b-R69TAG-His6 trep-DrrA(16-352)

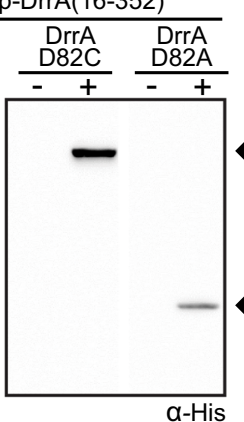

d

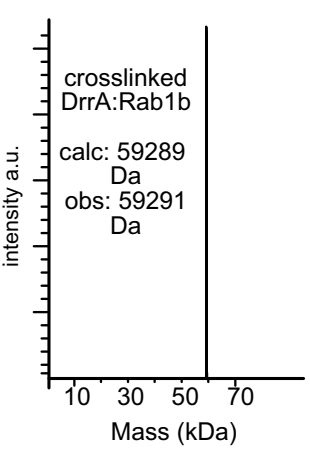

Mass $(\mathrm{kDa})$ i

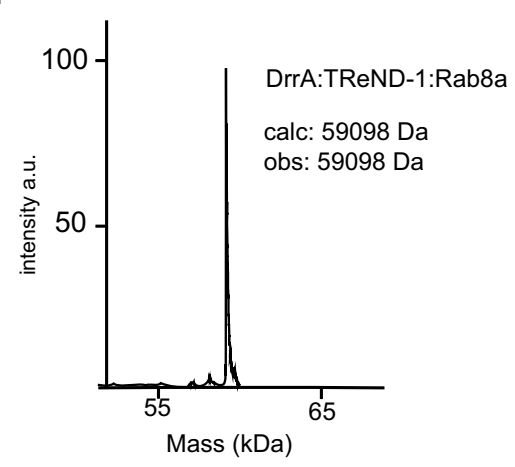

e

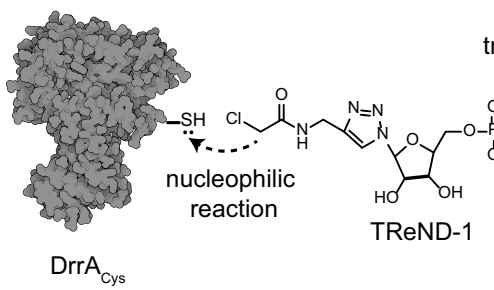

coomassie

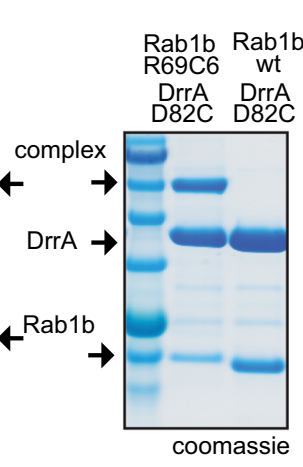

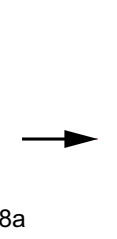

Rab1/Rab8a

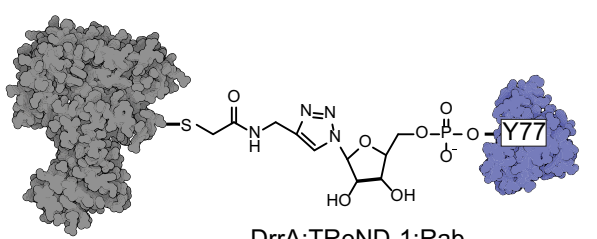

DrrA:TReND-1:Rab complex

f

g

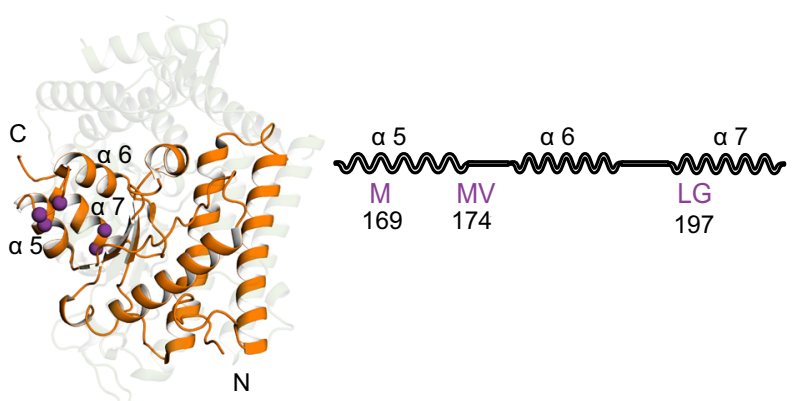

Fig. 1 Conceptual design for stabilising the low-affinity DrrAATase:Rab complex. a Schematic representation of domain organisation in full-length DrrA. b Covalent stabilisation of DrrA:Rab using site-specifically incorporated UAAs bearing bromoalkyl moieties (BrC6K). c $\alpha$-His6 western-blotting analysis of DrrA:Rab1b complex formation in living E. coli cells and SDS-PAGE analysis of in vitro DrrA:Rab1b complex formation. $\mathbf{d}$ Intact MS analysis of thioether linked AMPylated Rab1b:DrrA $282 C$ complex (a.u.: arbitrary units). e Trapping the DrrA:Rab complex via thiol-reactive nucleotide analogues. $\mathbf{f}$ Selection of Cys substitution sites (M169, M174, V175, L197 and G198, purple) in the model, and superimposition of DrrA 8 -218 (PDB: 3NKU, orange) onto GS-ATase (PDB: 3K7D, light green background). $\mathbf{g}$ SDS-PAGE shift analysis of DrrA:TReND-1:Rab1b complex formation. $\mathbf{h}$ SDS-PAGE shift analysis of preparative DrrA:TReND-1:Rab1b (DR1)/Rab8a (DR8) complex formation. i Intact mass spectrometry analysis of the DrrA:TReND-1:Rab8a complex. Source data are provided as a Source Data file.

vicinity of the catalytically important amino acids D110 $\operatorname{DrrA}$ and D112 DrrA $^{13}$ but rather on a surface patch located opposite to the active site of DrrA $\mathrm{A}_{\mathrm{ATase}}$, indicating either complete rearrangement of the previously characterised DrrA fold or an alternative mechanism for enzyme activation. Importantly, full-length D82 $\mathrm{C}_{\text {DrrA }}$ including the GEF and P4M domains (amino acids 1 -647; DrrA ${ }_{1-647}$ ) also displayed efficient crosslinking upon coexpression of R69BrC6K $\mathrm{K}_{\mathrm{Rab} 1 \mathrm{~b}}$ (Supplementary Fig. 1). In order to gain structural insight into Rablb binding to $\operatorname{DrrA}_{\mathrm{AT} \text { ase, we }}$ generated several milligrams of thioether-crosslinked DrrA 16-352: : $\mathrm{Rab}_{1} \mathrm{~b}_{3-174}$ complex and subjected them to crystallisation trials.

In parallel, we used a complementary in vitro crosslinking approach to stabilise the low-affinity DrrA $_{\text {ATase }}:$ Rab interface using our recently developed thiol-reactive nucleotide derivatives (TReNDs) as bridging functionalities ${ }^{24}$ (Fig. 1e). TReNDs carry a thiol-reactive chloroacetamide function that can covalently tether 
to a strategically placed cysteine in a proximity-enabled manner. In this case, chloroacetamide is positioned at an ATP derivative in which the nucleobase is replaced by a triazole ring. Thus, the combined reaction of the chloroacetamide with a strategically introduced cysteine in DrrA and the AMP transfer by DrrA to $\mathrm{Rab}$ proteins enables the formation of a covalently trapped complex between the enzyme and its target protein. Inspired by the identification of $\mathrm{D} 82 \mathrm{C}_{\mathrm{DrrA}}$ as a site for crosslinking with BrC6K-bearing Rablb (see above) and by a previous prediction of the ATP-binding site in DrrA ${ }^{25}$, we intended to probe both the putative adenine binding pocket and the amino acid residues located at the opposite surface of DrrA for complex formation with TReNDs and Rablb. Therefore, we generated $\operatorname{DrrA}_{16-352}$ constructs bearing M169C, M174C, V175C (the putative adenine interaction interface) and L197C, and G198C (opposite to the putative adenine interaction site) substitutions (Fig. 1f), and tested their ability to form a covalent complex with TReNDs and $\mathrm{Rab} \mathrm{b}_{3-174}$ loaded with the non-hydrolysable GTP analogue GppNHp. TReND-1 was indeed transferred by wt DrrA 16-352 $_{20}$ to Rablb $b_{3-174}$, indicating that it is correctly positioned in the catalytic centre (Supplementary Fig. 3). Using recombinantly purified proteins, TReND-1 successfully formed a covalent ternary complex with $\mathrm{Rablb}_{3-174}$ and $\operatorname{DrrA}_{16-352}-\mathrm{L} 197 \mathrm{C}$, as demonstrated by an apparent increase in molecular weight in denaturing SDS-PAGE (Fig. 1g), while ATP failed to form a covalently linked ternary complex, as expected. The reaction was site-specific since other mutants did not produce a covalent product (Fig. $1 \mathrm{~g}$ and Supplementary Fig. 4). Importantly, residue L197 Drra is also not located in the vicinity of the ATase active site; it is situated on the same surface patch as residue D82 DrrA opposite the catalytically active amino acid residues of DrrA (Supplementary Fig. 1).

Encouraged by the efficiency of our crosslink formation with the DrrA $_{16-352}$-L197C mutant, we attempted to produce larger quantities of the ternary DrrA-TReND-1-Rablb complex for structural investigations by X-ray crystallography. Several milligrams of pure DrrA 16-352:TReND-1:Rablb $_{3-174}$ complex were obtained, but the resulting crystals diffracted only poorly as it was the case for the thioether crosslinked $\operatorname{DrrA}_{16-352}: \operatorname{Rab}_{1} b_{3-174}$ described above. Therefore, we also generated the TReND-1linked complex with the GTPase domain of the close Rablbhomologue Rab8a (amino acids 6-176; Rab8a $\mathrm{a}_{6-176}$; Fig. 1h). Rablb and Rab8a share 53\% sequence identity, their structures can be superimposed with an RMSD (root-mean-square deviation) of about $0.5 \AA$, and Rab8a can be AMPylated by DrrA in vitro with very similar kinetics (Supplementary Fig. 5) ${ }^{13}$. The integrity, homogeneity, and correctness of the covalently linked ternary DrrA:TReND-1:Rab8a complex were validated using highresolution (HR) MS of intact proteins, and the results demonstrated excellent accordance between experimental and theoretical mass (Fig. 1i). Thus, combining thiol-reactive UAAs or nucleotides with cysteine-substituted DrrA allowed us to prepare covalent DrrA-Rab complexes, overcoming the difficulties in accessing the transient DrrA $_{\text {ATase }}-\mathrm{Rab}$ complex by more traditional approaches.

Structure of the DrrA:Rab8a complex. In order to obtain insight into the molecular basis of DrrA substrate recognition, we determined the crystal structure of the DrrA $16-352$ :TReND-1: $\mathrm{Rab8a}_{6-176}$ complex bound to GppNHp at $2.1 \AA$ resolution (DrrA 16-352:TReND-1:Rab8a $\mathrm{a}_{6-176}$ will be referred to as DrrA: Rab8a hereafter for clarity) (Fig. 2a, Supplementary Table 2 and Supplementary Fig. 6). The complex binding mode observed in the crystal structure was unexpected since the Rab molecule does not interact with the catalytic centre of DrrA $\mathrm{A}_{16-352}$ constituted by the catalytically important residues D110
Instead, Rab8a:GppNHp binds to a surface patch opposite the catalytic centre that we refer to as the non-conventional (NC) Rab binding site (RBS), hereafter referred to as NC-RBS (Fig. 2a, d). The complex exhibits the general structural features of the previously determined individual subunits of Rab8a:GppNHp, and parts of DrrA $\mathrm{A}_{8-218}$ and $\operatorname{DrrA}_{210-534}$ (Fig. 2a) ${ }^{13,17,26}$. In accordance with previously solved structures, Rab8a in complex with DrrA has a fold consisting of a central six-stranded $\beta$-sheet surrounded by five $\alpha$-helices ${ }^{26}$. Albeit individual crystal structures of $\mathrm{N}$ - and C-terminal parts of DrrA are available ${ }^{13,17}$, the structure of the full ATase domain of DrrA has not been reported to date. The general arrangement of the secondary structure elements of the so far solved fragments agrees very well with the full ATase domain, yet significant positional differences in the catalytic site residues were observed. Based on the structures of other ATases belonging to the DNA polymerase $\beta$-like enzyme family, the catalytic motif of DrrA is expected to be G98-S99-L100-X 11 -D110-X-D112 2 DrrA, with D110 DrrA and D112 DrrA involved in coordinating the essential magnesium ion. In contrast to the previous $\operatorname{DrrA}_{8-218}$ fragment structure ${ }^{13}$, the position of D110 ${ }_{\text {DrrA }}$ and D112 $2_{\text {DrrA }}$ is well defined and the residues appear to be capable of $\mathrm{Mg}^{2+}$ coordination (Fig. 2a).

The covalently bound TReND-1 is well defined in the electron density in the complex crystal structure (Fig. 2b). However, due to structural differences of the chemical linker, bridging the TReND1 ribose and C197 in DrrA, compared to ATP, only few interactions with the central scaffold of the linker are formed, which is reflected by the higher B-factors in respect to the surrounding amino acid residues (Supplementary Fig. 7). In the DrrA:Rab8a complex structure, switch I (E30 Rab8a, F33 Rab8a and N34 $4_{\text {Rab8a }}$ ), the interswitch region (D44 $4_{\text {Rab8a }}$ and K58 $8_{\text {Rab8a }}$ ), and switch II (Q60 Rab8a and R69 Rab8a $_{\text {a }}$ ) of Rab8a mainly interact with the N-terminal half of the ATPase domain of DrrA opposite the catalytic motif, centred at amino acids R70 DrrA, Q71 $1_{\text {DrrA }}$ and K74 ${ }_{\text {DrrA }}$. Evaluation using the Protein Interfaces, Surfaces and Assemblies (PISA) web service ${ }^{27}$ showed that the solventaccessible area buried upon complex formation is small (648 $\AA^{2}$ ), indicating that the complex is of low affinity and requires covalent linkage in order to be observed (Fig. 2c; hydrophobic interactions can be seen in Supplementary Fig. 8). In the previous structure of the $\mathrm{N}$-terminus $\mathrm{DrrA}_{\mathrm{AT} \text { ase, }}$ the catalytic site (D110 DrrA, D112 $2_{\text {DrrA }}$ and D150 DrrA $)$ was disordered and incompetent for $\mathrm{Mg}^{2+}$ coordination in this arrangement. However, in our complex crystal structure, all three acidic amino acids are properly positioned upon Rab binding to the NC-RBS, as observed by superimposition with the ATase-domain of glutamine synthetase adenylyl transferase from E. coli ${ }^{18}$ (Fig. 2e).

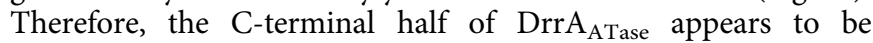
important for overall structural integrity and correct alignment of the catalytic site.

The structure of free Rab8a:GppNHp superimposes very well with Rab8a in the NC-RBS-complex, with an RMSD of $0.38 \AA$, indicating that binding of Rab8a to DrrA does not result in global structural changes in Rab8a (Fig. 2f) ${ }^{26}$. Similarly, AMPylated Rab1b also superimposes with Rab8a in the NC-RBS-complex, with an RMSD of about $0.6 \AA$, demonstrating that binding to the NC-RBS of DrrA is also unlikely to induce significant conformational changes in Rab1b (Fig. 2f) ${ }^{13}$.

Consequently, the DrrA:Rab8a crystal structure reveals a previously unrecognised NC-RBS opposite the catalytic centre of the ATase.

Validation of the non-conventional Rab-DrrA interface. In order to rule out the possibility that binding of Rab1b or Rab8a to the NC-RBS is an artefact of covalent linkage caused by TReND-1, 
a

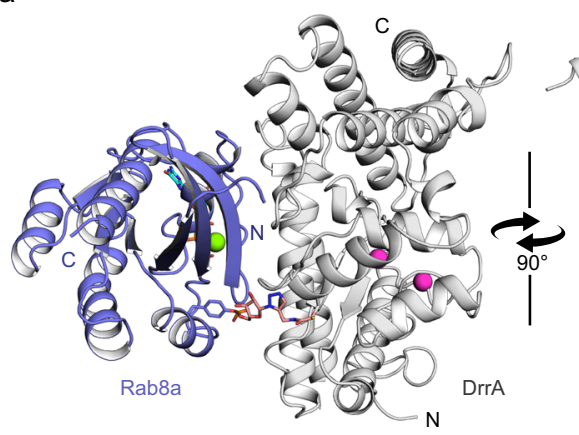

C

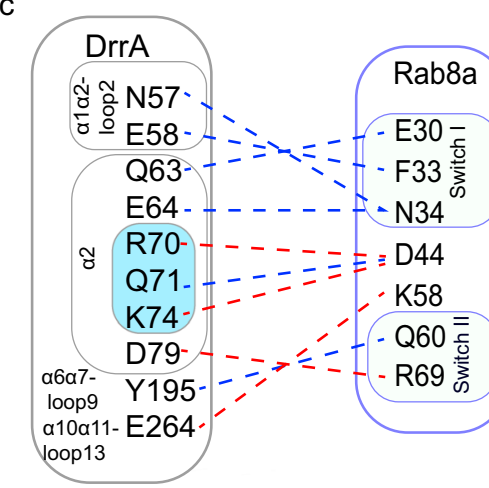

e

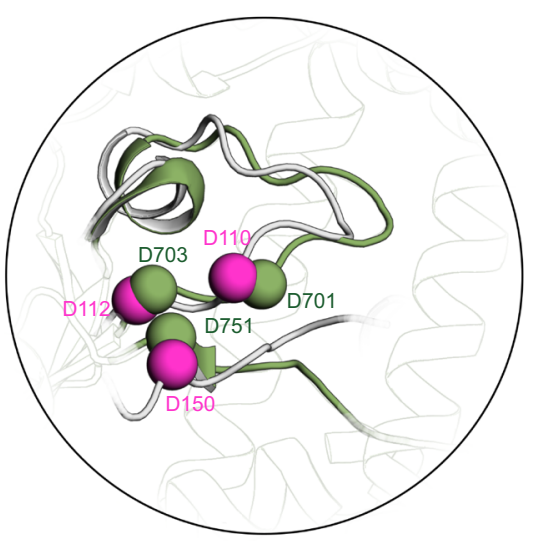

b

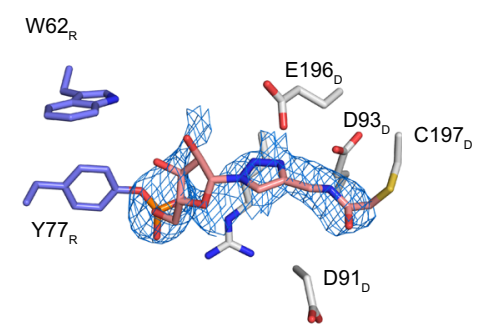

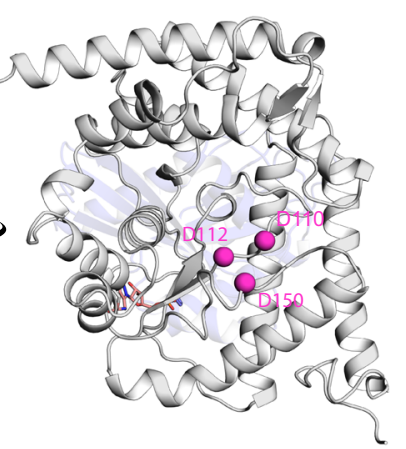

d

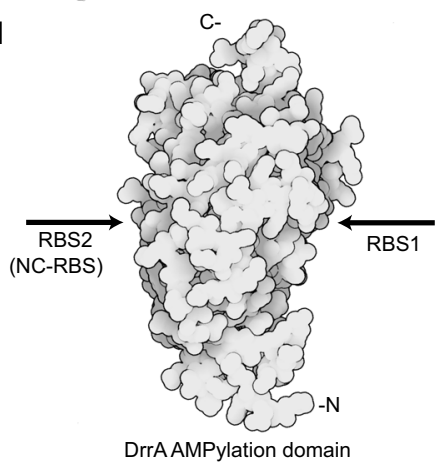

DrrA AMPylation domain

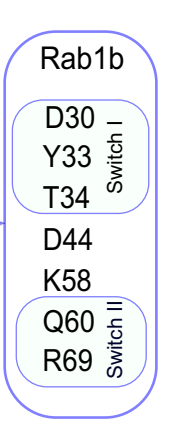

f

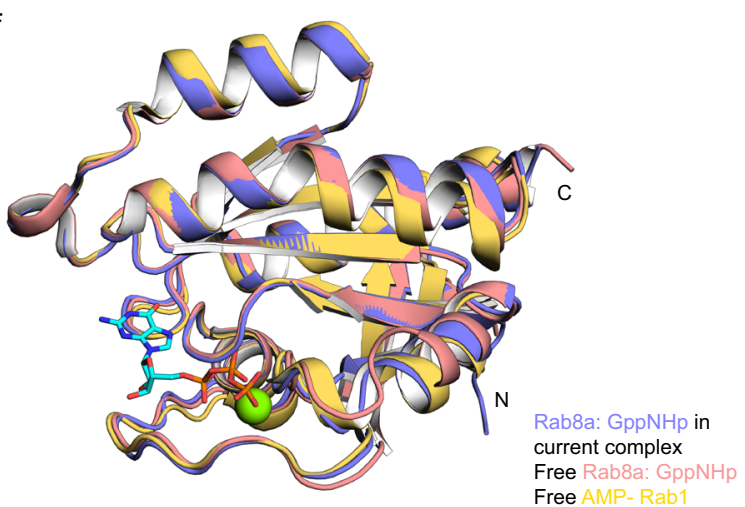

Fig. 2 Structure of the DrrA:Rab8a complex. a Orthogonal views of the Rab8-DrrA complex. Pink spheres denote the catalytic Asp residues of DrrA. The green sphere represents the $\mathrm{Mg}^{2+}$ ion. $\mathbf{b}$ Linker density from the unbiased simulated-annealing omit DFo-Fc electron density map contoured at $2.5 \sigma$. The $\mathrm{R}$ subscript denotes Rab8a, and the D subscript denotes DrrA. c Schematic representation of the Rab8-DrrA interface. Interactions are shown with dashed lines; hydrogen bonds are blue and salt bridges are red. The corresponding interaction residues in Rab1 are shown in the panel on the right. Important residues for maintaining enzymatic activity are coloured cyan, and ' $\alpha$ ' represents $\alpha$-helix. $\mathbf{d}$ Demonstration of the conventional site (RBS1, containing the catalytic centre) and non-conventional site (RBS2, the back face of the catalytic centre. e Structural comparison between the catalytic centre in GS-ATase (PDB: 3K7D, green) and the catalytic centre in DrrA in the DrrA-Rab8a complex. The catalytic centre of DrrA includes D110 DrrA, D112 ${ }_{\text {DrrA }}$ and D150 DrrA. The catalytic centre of GS-AT includes D701 GS-AT, D703 GS-AT $_{\text {and D753 }}$ GS-AT. $\mathbf{f}$ Structural superposition of free AMP-Rab1:GppNHp (PDB: 3NKV, yellow) and Rab8a (PDB: 4LHW, pink) with Rab8a (blue) from the complex with DrrA. Green spheres indicate $\mathrm{Mg}^{2+}$ ions, and GppNHp is shown in stick representation.

we validated the observed complex structure using complementary approaches. First, we produced alanine mutants of DrrA amino acids involved in the interface with Rab8a. Since the Rab-proteins can interact with the NC-RBS and the catalytic site of DrrA $A_{\text {ATase }}$ at the same time (Fig. 2d), a distinction of the effect of Rab-mutations on AMPylation by binding to either site would not be possible. Hence, we only included DrrA alanine mutations for subsequent experiments. To assess whether the alanine substitutions impaired the general stability of DrrA, we subjected them to thermal unfolding and monitored the change in circular dichroism (CD) signal. Compared with the melting point $\left(T_{\mathrm{m}}\right)$ of wt DrrA $\left(T_{\mathrm{m}}=60.1^{\circ} \mathrm{C}\right)$, the other alanine substitutions, but not $\mathrm{D} 9 \mathrm{~A}_{\text {DrrA }}$, displayed mild changes with an amplitude from 0.1 to $2.5^{\circ} \mathrm{C}$, demonstrating that these mutants are biochemically similar to wt DrrA $16-352$ (Fig. 3a). D79 $9_{\text {DrrA }}$ forms intramolecular polar interactions with $\mathrm{K} 36_{\text {DrrA }}$ and Y40 DrrA in the complex crystal structure, and $\mathrm{D} 79 \mathrm{~A}_{\text {DrrA }}$ destabilised DrrA, as evidenced by a decrease of $4.2{ }^{\circ} \mathrm{C}$ in the melting point.

Since the complex was obtained with Rab8a as a Rablbsurrogate, we tested whether there are notable differences of the mode of DrrA-binding. Structural superimposition of Rab1b with Rab8a from the DrrA:Rab8a complex revealed four amino acids 
a

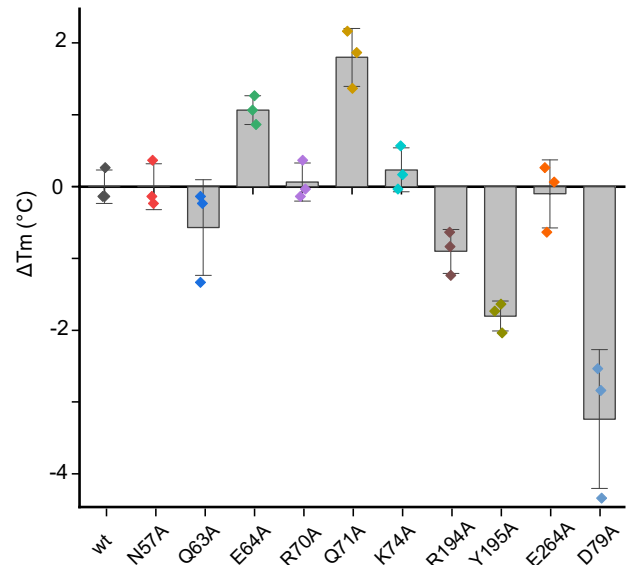

b

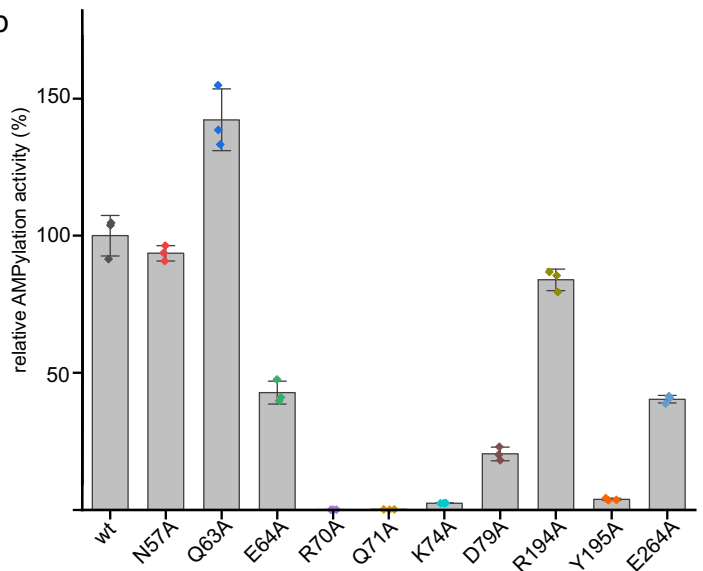

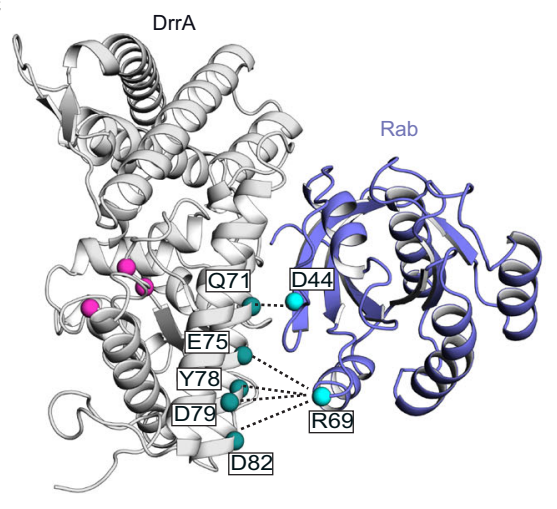

d

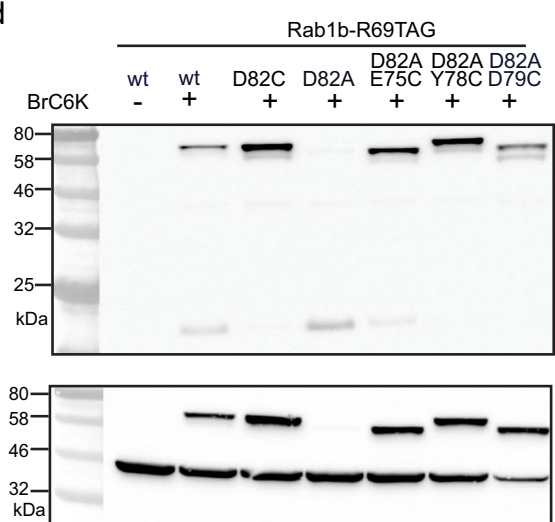

Rab1b-D44TAG

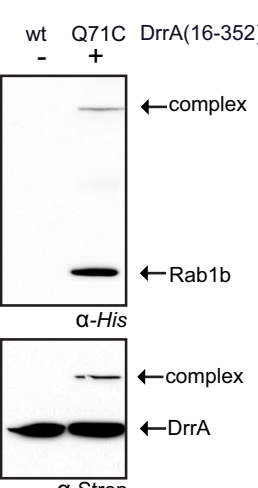

Fig. 3 Confirmation of the NC-RBS complex interface. a Melting point (Tm) determination of DrrA and DrrA alanine mutants by circular dichroism (CD). The melting point of wt DrrA is $60.1^{\circ} \mathrm{C}$. Data are means \pm standard error of the mean (SEM) from three independent experiments. $\mathbf{b}$ Determination of Rab1b-AMPylation rates in vitro by DrrA with mutations in the non-conventional site. The $k_{\text {cat }} / K_{M}$ value of wt DrrA is $8.0 \times 10^{5} \mathrm{M}^{-1} \mathrm{~s}^{-1}\left( \pm 2.0 \times 10^{4} \mathrm{M}^{-1} \mathrm{~s}^{-1}\right)$. Data are means \pm standard error of the mean (SEM) from three independent experiments. c Representation of selected interactions for in cellulo crosslinking in E. coli. Pink spheres are the catalytic Asp residues of DrrA. d Pairwise BrC6K (within Rab1b) and Cys mutations (within DrrA) based on the DrrA:TReND-1:Rab complex lead to covalent crosslink formation, if the BrC6K-based thioether linker is able to bridge the Rab1b-DrrA interface, as observed by $\alpha$-His 6 and $\alpha$-Strep western-blotting. Source data are provided as a Source Data file.

that are located in the interface and differ between the Rabproteins $^{13}$ : L26, A29, T32, E35 vs. Rab8a: F26, S29, A32, S35. However, none of these amino acids are involved in interactions in the complex interface and do not create steric clashes that could impair complex formation between $\operatorname{DrrA}_{16-352}$ and Rablb (Fig. 2c and Supplementary Fig. 9). Consistent with the lack of steric clashes and the high sequence homology between Rablb and Rab8a, the AMPylation rates of these GppNHp-loaded Rabs by DrrA are comparable (Supplementary Fig. 5). Thus, the mode of binding of DrrA ${ }_{16-352}$ to Rablb:GppNHp and Rab8a:GppNHp is identical.

Next, we investigated the AMPylation activity of these DrrA mutants toward GppNHp-bound Rablb and Rab8a. AMPtransfer results in the decrease of GTPase tryptophan fluorescence and thus can be used for monitoring AMPylation as reported previously ${ }^{14}$. The DrrA mutants $\mathrm{D} 79 \mathrm{~A}_{\text {DrrA }}, \mathrm{Y} 195 \mathrm{~A}_{\text {DrrA }}$ and K74A $\mathrm{A}_{\text {DrrA }}$ exhibited a 5 -fold, a 30 -fold and a 50 -fold decrease in activity toward Rab1, respectively, and R70A $A_{\text {DrrA }}$ and Q71A $A_{\text {Drra }}$ displayed at least 1000 -fold activity reduction toward Rab1 (Fig. 3b and Supplementary Fig. 10). The strong decrease in AMP transfer rates can be explained by an ionic and polar interaction network involving R70 DrrA, Q71 $1_{\text {DrrA }}$ and K74 DrrA with D44 $4_{\text {Rab1b/Rab8a. }}$ Although Rab8a was used as a surrogate of Rab1b for determining the complex structure, the kinetics of Rab8a-
AMPylation by the DrrA-mutants yields similar results, confirm-

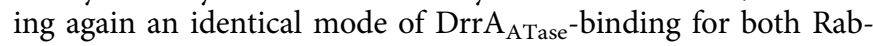
proteins (Supplementary Figs. 5 and 10). Therefore, these results suggest that the NC-RBS may play a significant role in regulating DrrA AMPylation activity.

In order to further validate our results, we envisioned testing the complex interface in cellulo using proximity-enabled crosslinking of BrC6K-bearing Rab1b and Cys-mutants of DrrA. Guided by the complex structure, we selected additional combinations for pairwise BrC6K- (within Rab1b) and Cyssubstitutions (within DrrA) that we predicted to result in sitespecific covalent crosslinking when co-expressing the constructs in E. coli. This approach allowed us to screen the interface of the complex within a distance of $13 \AA$. We confirmed the selective formation of thioether crosslinks between BrC6K incorporated site-specifically at position $69_{\text {Rablb }}$ within Rab1b and Cys residues introduced at E75 DrrA, Y78 ${ }_{\text {DrrA }}$ and D79 DrrA within DrrA in living E. coli cells using SDS-PAGE, $\alpha$-His6 and $\alpha$-Strep westernblotting analyses (Fig. 3c, d and Supplementary Fig. 11). Likewise, crosslinks were observed between D44BrC6 $\mathrm{K}_{\mathrm{Rab} 1 \mathrm{~b}}$ and Q71C $\mathrm{CrrA}_{\mathrm{D} A}$, as well as between D31BrC6 $\mathrm{K}_{\text {Rab1b }}$ and E280 DrrA of wt DrrA. Importantly, the pairwise introduction of $\mathrm{BrC}_{\mathrm{Rab} 1 \mathrm{~b}}$ and cysteines $_{\text {DrrA }}$ at more remote positions $(>15 \AA)$ did not result in covalently linked complexes, indicating that the crosslinking 
reaction is specific for proximal sites that can be spanned by the flexible bridge formed between $\mathrm{BrC6K}$ and nucleophilic residues (Supplementary Fig. 11).

In summary, our mutational analyses and covalent complex formation studies demonstrate that the interaction of Rablb with the NC-RBS of DrrA is related to the AMPylation activity of the enzyme, and not an artefact of covalent complex formation using TReND-1 or the UAA BrC6K.

Rab1-binding to the NC-RBS activates DrrA. Even though we confirmed the presence of an NC-RBS in the complex crystal structure, the functional relevance of Rab1b binding to the noncatalytic site remains unclear. We first explored whether Rablb binding causes structural changes in DrrA. Since the apo structure of DrrA has not yet been determined, superimposition of the previously reported $\mathrm{N}$ - and $\mathrm{C}$-terminal parts $\left(\mathrm{DrrA}_{8-218}\right.$ and $\mathrm{DrrA}_{218-340}$, referred as part- $\mathrm{N}$ and part-C respectively hereafter) of the enzyme on our complex crystal structure served as a relevant structural model (Fig. 4a) ${ }^{13,17}$. Even though part-C and part- $\mathrm{N}$ have been structurally characterised separately, they do not appear to be distinct functional units. Rather, intimate contacts between part- $\mathrm{N}$ and - $\mathrm{C}$ are present, even in the loop spanning amino acids, which links these two units. Also, ATasefragments lacking part-C or portions thereof do not have AMPylation activity and do not cause AMPylation-mediated cellular toxicity. Instead, part-C interacts with helix $\alpha 3$ and the loop a3- $\beta 2$, which is localised in the catalytic centre of the ATase loop (Supplementary Fig. 12), suggesting that these interactions are necessary for correct folding of this region and positioning of catalytically relevant amino acids. This view is supported by the fact that most interactions (nine out of ten amino acids) between DrrA $_{16-352}$ with the Rab-protein are formed by part-N (Supplementary Fig. 12 and Fig. 2c). The only amino acid interacting with the Rab from part-C is E264 DrrA $_{\text {and }}$ and mutation to alanine impairs the AMPylation activity moderately (Fig. 3b). Therefore, the lack of enzymatic activity of constructs lacking part-C cannot be explained by the deletion of essential amino acids involved in Rab-binding. Instead, this fact supports the notion that part-C stabilises regions in part- $\mathrm{N}$ necessary for catalysis.

Albeit no global structural differences between the composite putative apo structure and the full ATase domain occur (Fig. 4b), significant structural differences can be observed for the secondary structure elements in the vicinity of the $\operatorname{DrrA}_{\mathrm{ATase}}$ active site (Fig. 4c). Indeed, upon Rab binding, helix a 3 may be pulled into the protein core, and D110 DrrA $(\beta 2), D 112_{\text {DrrA }}(\beta 2)$ and D150 DrrA $(\alpha 4-\alpha 5$ loop) move together so that the active $\mathrm{Mg}^{2+}$ coordinating function of these amino acid residues can be fulfilled. Of note, we previously reported that AMPylationcompetent $\operatorname{DrrA}_{8-533}$ is not able to bind ATP in the absence of Rab125. Therefore, Rab binding to the NC-RBS of DrrA may lead to rearrangement of the enzyme active site. We therefore wondered whether Rab binding to the NC-RBS may regulate DrrA's AMPylation activity by reordering the catalytic residues. In order to investigate the potential influence of Rabib binding to the ATase domain of DrrA (i.e. $\operatorname{DrrA}_{16-352}$ ), we analysed the rate of Rablb AMPylation in dependence on the absolute concentration of active Rablb:GppNHp by measuring the decrease in Rab tryptophan fluorescence upon AMP modification. We observed a lag-phase in the AMPylation rate at low Rablb:GppNHp concentrations, indicative of a stimulatory contribution of Rablb binding at higher concentrations (Fig. 4d). Therefore, fitting the data to a simple Michaelis-Menten model (i.e. to a hyperbolic function) was not feasible; hence, a sigmoidal Hill-type function was instead employed. The cooperativity parameter resulting from the Hill fit was greater than $1(n=1.7 \pm 0.16)$, indicating a major contribution of Rablb:GppNHp-binding to the rate of AMPylation. DrrA $\mathrm{A}_{16-647}$ containing all three domains shows an identical kinetic profile $(n=1.6 \pm 0.20)$ (Fig. 4e).

Furthermore, we also tested the stimulatory function of Rablb binding to DrrA in cellulo in order to exclude the possibility of in vitro artefacts. Since DrrA causes AMPylation-induced cellular cytotoxicity in mammalian cells upon overexpression, the activity of individual DrrA mutants can be determined by quantifying the cell viability ${ }^{13}$. To this end, eukaryotic H1299 cells were transfected with the previously characterised alanine substitutions $\left(\mathrm{R} 7 \mathrm{~A}_{\text {DrrA }}, \mathrm{Q} 71 \mathrm{~A}_{\text {DrrA }}\right.$ and $\left.\mathrm{K} 74 \mathrm{~A}_{\text {DrrA }}\right)$ of an N-terminally eGFP (enhanced green fluorescent protein)-tagged $\mathrm{DrrA}_{8-533}$ construct. DrrA $_{8-533}$ was employed since the presence of the GEF domain $\left(\mathrm{DrrA}_{340-533}\right)$ is required to produce active, GTP-loaded Rab1b in the cell. Positively transfected cells were collected by fluorescence-activated cell sorting (FACS) and used for cell viability analysis by the absorbance-based MTS (3-(4,5dimethylthiazol-2-yl)-5-(3-carboxymethoxyphenyl)-2-(4-sulfophenyl)-2H-tetrazolium) assay. $\operatorname{DrrA}_{8-533}$ but not the AMPylation-deficient D110A/D112 $\mathrm{A}_{\mathrm{DrrA}}$ mutant caused pronounced cytotoxicity ${ }^{13}$. However, H1299 cells transfected with $\mathrm{DrrA}_{8-533}$ or with these selected single alanine mutant $\mathrm{R} 7 \mathrm{~A}_{\mathrm{DrrA}}$, Q71 $A_{\text {DrrA }}$ or $\mathrm{K} 4 \mathrm{~A}_{\mathrm{DrrA}}$ showed similar cytotoxicity (Fig. 4f). Although kinetics indicated that single alanine mutants $\left(R 70 A_{\text {DrrA }}, Q 71 A_{\text {DrrA }}\right.$ or $\left.K 74 A_{\text {DrrA }}\right)$ dramatically decrease the enzyme activity in vitro, results from experiments in cellulo differ from in vitro studies probably due to the longer duration of the in vivo experiments. To make an approximate comparison, we investigated Rab1b-AMPylation by the DrrA single mutants R70A $A_{\text {DrrA }}, Q 71 A_{\text {DrrA }}$ or K74A $A_{\text {DrrA }}$ via intact high-resolution mass spectrometry at $72 \mathrm{~h}$ incubation time. Extended incubation times of the single mutants resulted in nearly quantitative AMPylation of Rablb (Supplementary Fig. 13). Structural analysis of the DrrA:Rab8a-complex indicated that these three residues can interact with each other to constitute a triangular network, which may stabilise the interaction with D44 from Rab1b/Rab8a. We therefore reasoned that the simultaneous combination of these mutations is required to result in a reduction in cytotoxicity compared to wt $\mathrm{DrrA}_{8-533}$. The triple mutant R70A/Q71A/ $\mathrm{K}_{74} \mathrm{~A}_{\text {DrrA }}$ (corresponding to the NC-RBS) of DrrA $\mathrm{A}_{8-533}$ was not cytotoxic (Fig. 4g). These data show that mutations in the NCRBS affect cellular AMPylation and DrrA-mediated cytotoxicity, indicating that the AMPylation activity of DrrA is regulated via Rablb:GppNHp binding to the non-catalytic site. To further prove that mutations in the NC-RBS site of DrrA occlude binding of Rab under physiological conditions and thereby affect DrrA activity, we set out to perform our UAA-based, proximitytriggered crosslinking approach in living HEK293T cells. We coexpressed BrC6K-bearing Rab1b (Rab1b-R69BrC6K) together with different non-toxic eGFP-DrrA ${ }_{8-533}$ variants in HEK293T cells. Crosslinking was specific for Rab1b-R69BrC6K and the previously identified D82C mutant of eGFP-DrrA ${ }_{8-533}$. In accordance with cytotoxicity data, the triple alanine mutant eGFP-DrrA $_{8-533}(\mathrm{R} 70 \mathrm{~A} / \mathrm{Q} 71 \mathrm{~A} / \mathrm{K} 74 \mathrm{~A})$ bearing a D82C mutation was deficient in forming a crosslinked complex with Rab1bR69BrC6K (Fig. 4h and Supplementary Fig. 14).

In addition, we wondered whether the presence of the AMP group in Rab1b at Y77 Rablb would exclude its binding to the NCRBS. We therefore utilised our established approach and monitored time-resolved crosslink formation (via D82 $\mathrm{C}_{\text {DrrA }}$ ) of either AMPylated or non-AMPylated Rab1b-R69BrC6K-His6 with the DrrA ${ }_{16-352}$-D82C-D110A-D112A variant by SDS-PAGE and western blotting. The AMPylation-deficient D110A/D112A DrrA-mutant was used to exclude modification of nonAMPylated Rablb during the experiment. Indeed, both AMPylated and non-AMPylated Rab1 form a crosslinked complex 
a

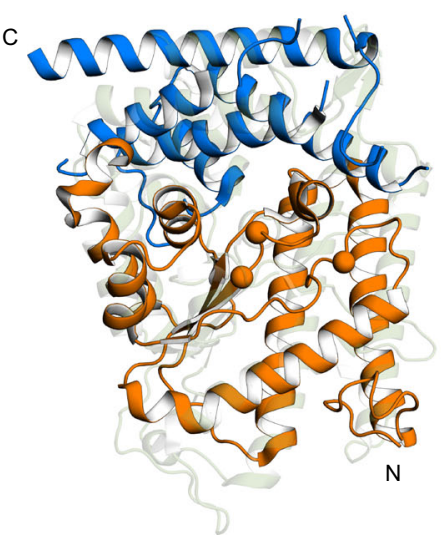

d

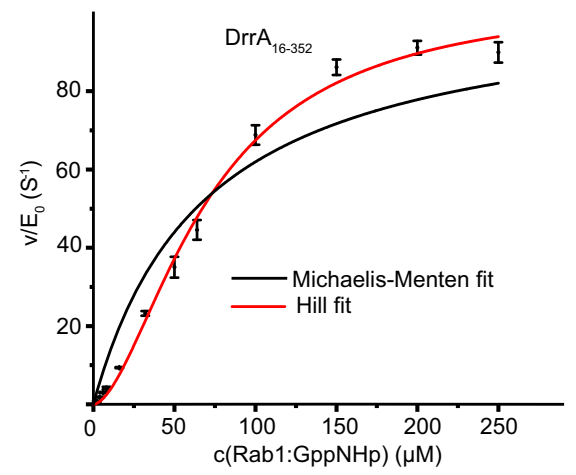

f

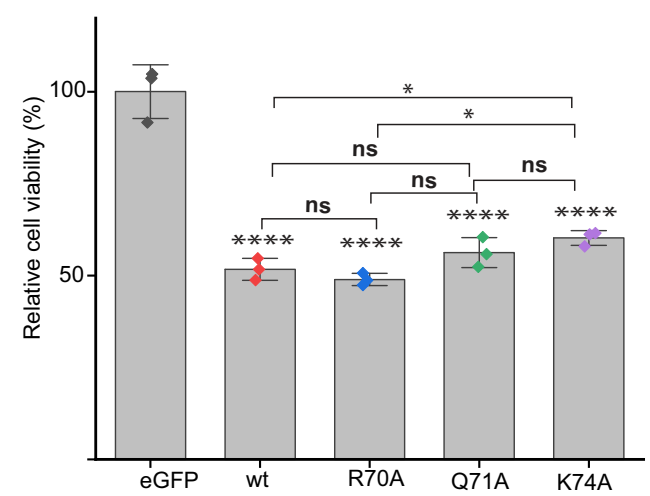

b

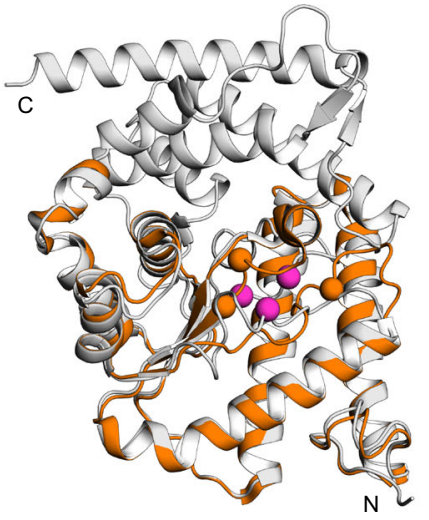

e
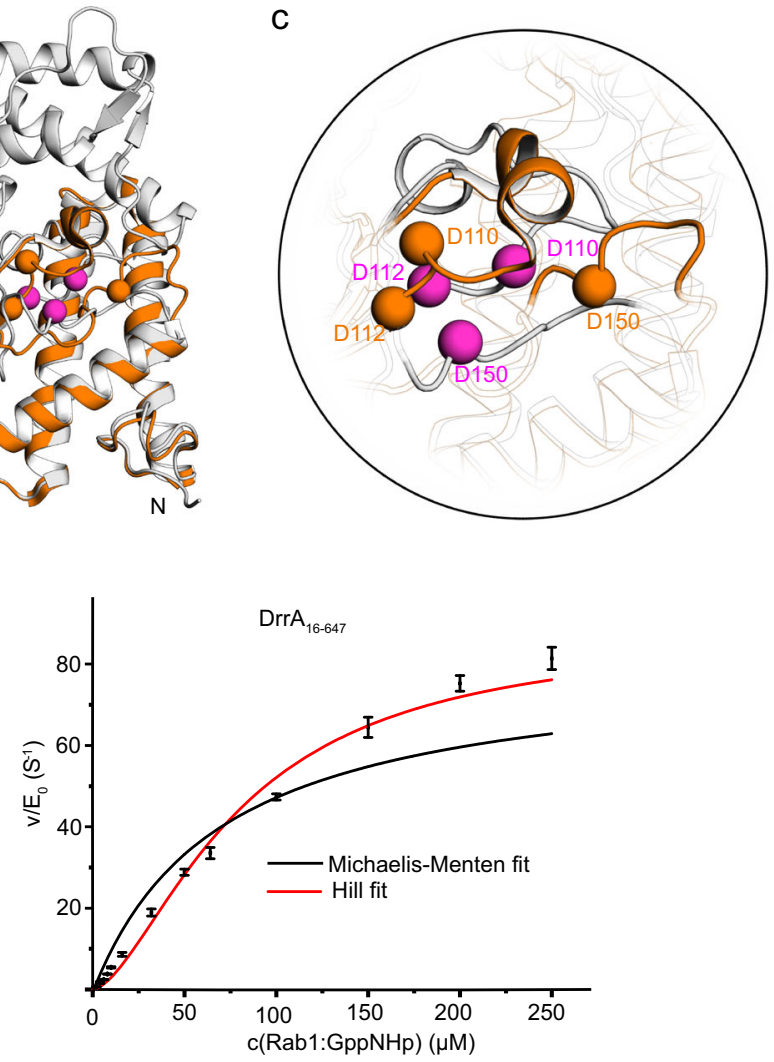

g
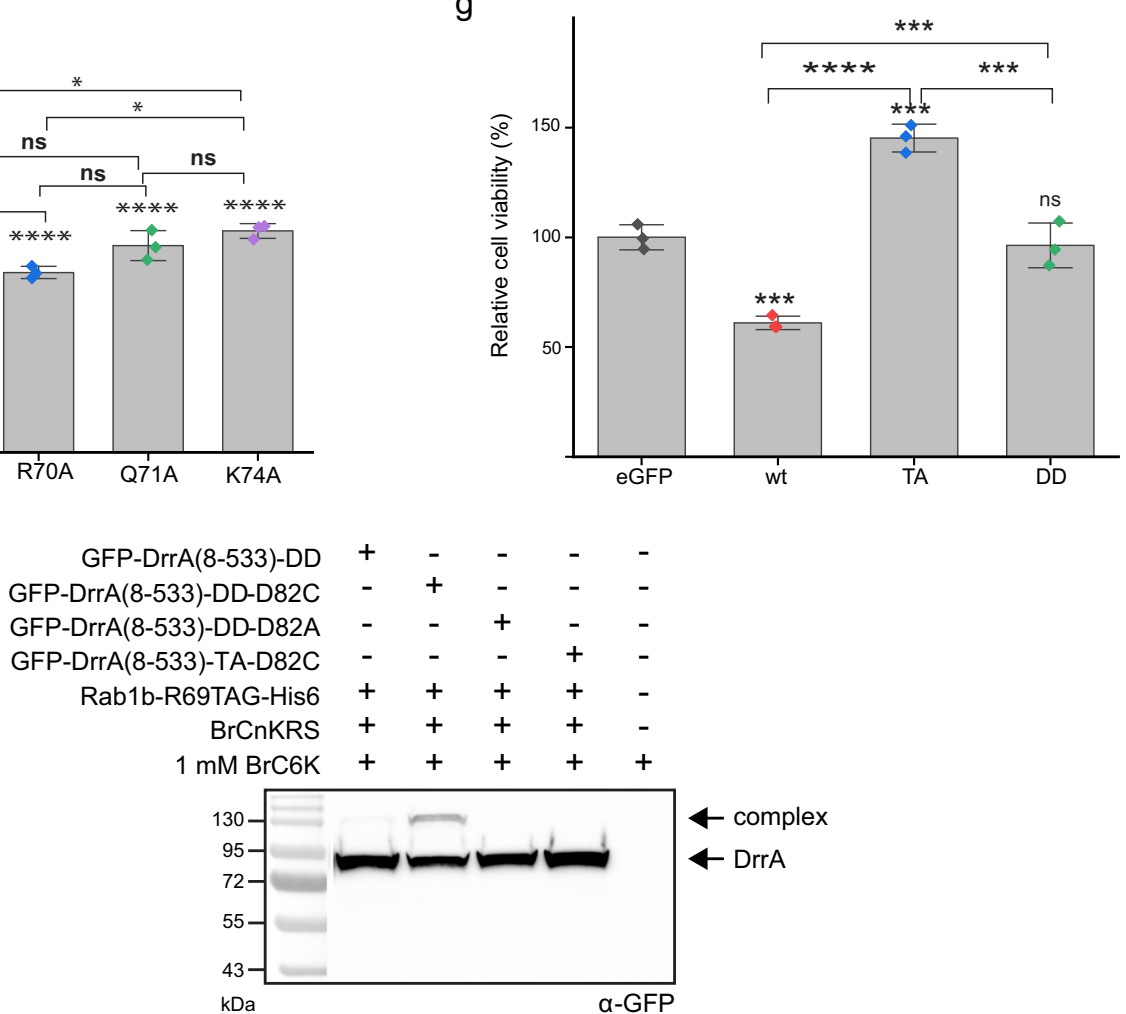

in vitro with minor differences in the rate of reaction (Supplementary Fig. 15). Hence, the presence of AMP in Rablb does not exclude binding to the NC-RBS.

In summary, biochemical experiments, cytotoxicity data and crosslinking approaches confirm that the AMPylation activity of DrrA is allosterically stimulated by Rab1b-binding to the NC-
RBS. However, to what extent the binding of Rablb to the NCRBS leads to a structural reorganisation of the catalytic site cannot be deduced entirely from the available structure comparisons: In the absence of the C-terminal part of the ATase-domain (i.e. part-C), essential intramolecular interactions between part- $\mathrm{C}$ and part- $\mathrm{N}$ are lacking and thus the catalytic 
Fig. 4 Allosteric activation of DrrA by active Rab1. a Construction of the DrrA apo-form. DrrA ${ }_{8-218}$ (PDB: 3NKU, orange) and DrrA $193-352$ (PDB: 3LOI, blue) are superimposed onto GS-ATase (PDB: 3K7D, light green background). Orange spheres represent the catalytic Asp residues of DrrA $8-218 . \mathbf{b}$ Global

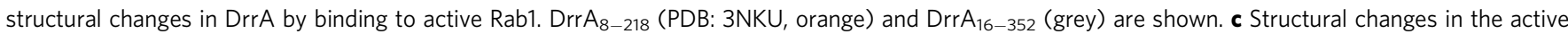
centre of DrrA induced by Rab1 binding to the non-conventional site. Purple spheres represent the catalytic centre of DrrA $A_{16-352}$ in the DrrA:Rab8a complex. Orange spheres represent the catalytic centre of $\operatorname{DrrA}_{8-218}$ (PDB: 3NKU, orange). d Sigmoidal dependence of AMPylation on the active Rab1 concentration. The red curve represents the Hill fit with a cooperativity parameter of $n=1.7 \pm 0.16$; the black curve is the Michaelis-Menten fit. Data are means \pm SEM from three independent experiments. e Full- length DrrA 16-647 -mediated AMPylation. The red curve represents the Hill fit with a cooperativity parameter of $n=$ $1.6 \pm 0.20$; the black curve is the Michaelis-Menten fit. Data are means \pm SEM from three independent experiments. $\mathbf{f}$ Cytotoxicity analysis of DrrA single alanine mutants in $\mathrm{H} 1299$ cells. Cell viability values (determined by MTS assay) of DrrA-expressing cells (eGFP-positive) were determined in relation to the eGFP vector control. WT: DrrA 8-533; R70A: DrrA 8-533_R70A; Q71A: DrrA 8-533_Q71A; K74A: DrrA 8-533_K74A. Data are means \pm SEM from three independent experiments. One-way analysis of variance (ANOVA) was applied; ns, $p>0.05$; ${ }^{\star} 0.01<p<0.05 ;{ }^{\star \star \star \star} p<0.0001$. Comparing to the wt, the $p$ values from R70A, Q71A, K74A are 0.9172, 0.6747, and 0.1594 respectively. The $p$ value between R70A and Q71A is 0.2671, the one between Q71A and K74A is 0.7575 , and the one between R70A and K74A is 0.0454. $\mathbf{g}$ Cytotoxicity analysis of DrrA mutants in H1299 cells. Cell viability values (determined by MTS assay) of DrrA-expressing cells (eGFP-positive) were determined in relation to the eGFP vector control. TA: DrrA 8 -533_R70A_Q71A_K74A; DD: DrrA8. 533_D110_D112A. Data are means \pm SEM from three independent experiments. One-way analysis of variance (ANOVA) was applied; ns, $p>0.05 ;{ }^{\star \star \star} p<$ $0.001 ;{ }^{\star \star \star} p<0.0001$. Comparing the eGFP, the $p$ values from wt, TA, and DD are 0.0005, 0.0002, and 0.9095, respectively. The $p$ value between wt and DD is 0.0010 . The $p$ value between TA and DD is 0.0001. h Proximity-triggered crosslinking between BrC6K-bearing Rab1b and eGFP-DrrA 8 -533 mutants in living HEK293T cells as observed by $\alpha$-GFP WB. Crosslinking is specific for DrrA-D82C and Rab1b-R69BrC6K for DD-DrrA variants; the corresponding triple alanine DrrA-variant (TA-DrrA) is deficient in crosslinking. Source data are provided as a Source Data file.

residues may be more flexible than in a-yet unavailable-ATase reference structure. Thus, it is possible that the impact of the here-observed structural changes introduced by Rab1b on the ATase-domain of DrrA are less pronounced in context of the fulllength DrrA protein.

Formation of a Rab-DrrA complex via the catalytic site. In addition to assessing the significance of the NC-RBS in DrrA, we attempted to create a complex with the Rab protein bound to the catalytic site of DrrA. For this purpose, we applied the same crosslinking strategy by combining TReND-1 with Cys substitutions located in close proximity to the putative ATP-binding site in DrrA $_{16-352}$. We produced the A176C, D177C and T181C variants of DrrA as potential sites for TReND-1 reaction since these amino acids are likely to be close to the ATP nucleobase interaction site (Fig. 5a). Indeed, DrrA $16-352$ containing the A176C substitution, but not D177C or T181C, formed a covalent ternary complex with $\mathrm{Rab} \mathrm{b}_{3-174}$ in vitro, as indicated by an increase in molecular weight observed by SDS-PAGE (Fig. 5b). Using this approach, we were able to obtain milligram amounts of the ternary DrrA $16-352-A 176 C$ :TReND-1:Rab1b $3-174$ complex with high purity, as demonstrated by intact HR-MS (Fig. 5c).

Structure determination of the complex failed due to a lack of diffracting crystals. Nevertheless, the ternary complex from the catalytic site of DrrA permitted us to further validate the presence of NC-RBS of DrrA by evaluating and comparing the catalytic activity. For this purpose, we compared the relative AMPylation activity of DrrA $\mathrm{A}_{16-352}$ with the ternary complexes linked via the NC-RBS and the catalytic site using the time-resolved change in tryptophan fluorescence of Rab1b in response to AMPylation (Fig. 5d). As expected, the DrrA:Rab1 complex linked via the NCRBS stimulated the AMPylation activity compared with free DrrA $_{16-352}$. By contrast, the complex linked via the catalytic site was unable to AMPylate Rab1b, showing that the presence of the covalently linked Rab1b blocks the access of substrate molecules. Therefore, our results imply that $\operatorname{DrrA}_{16-352}$ contains two separate Rab-binding platforms. Hence, we propose that allosteric binding of active Rab1 to the NC-RBS switches the active site of DrrA $_{\text {ATase }}$ from an unstructured AMPylation-deficient state to an organised AMPylation-competent state, which further mediates AMPylation activity (Fig. 5e).

\section{Discussion}

Infection of eukaryotic cells by L. pneumophila is a coordinated process characterised by the controlled release and activity regulation of bacterial proteins. The different enzymatic activities of DrrA (GEF and AMPylation) appear to be influenced in particular by the secretion of the protein during the early phases of infection, and the regulated localisation at the LCV ${ }^{28}$. However, in addition to the AMPylation of Rabl by the N-terminal ATase domain, DrrA can also modify a number of other Rab GTPases, which could cause high cytotoxicity in the case of excessive and mislocalised activity ${ }^{13}$. To avoid these undesirable side effects, which potentially could diminish the chances of an effective infection, additional direct control of the AMPylation activity of DrrA in L. pneumophila may be important. Using two different proximity-triggered and site-specific chemical crosslinking approaches employing UAAs bearing alkylbromide moieties or thiol-reactive ATP-derivatives combined with cysteinesubstituted DrrA-variants, we discovered a non-conventional Rab1-binding site (NC-RBS) in DrrA. Also, the structure of the DrrA $_{\mathrm{A} \text { Tase }}$ permits us to produce a composite structural model by superimposing previous structures ${ }^{13,17}$, in which three different Rab1 binding sites can be visualised (Fig. 5e). The NC-RBS profoundly contributes to the regulation of the AMPylation activity of DrrA in vitro: GTP-loaded Rablb binds to an allosteric site located opposite to the catalytic centre of the AMPylation domain and stimulates AMP transfer to the GTPase. Thus, two molecules of Rab1b:GTP simultaneously bind to DrrA: one to the allosteric NC-RBS, the other to the catalytic AMPylation site. Hence, DrrA binds to the LCV by virtue of the PI4P binding P4M and catalyses the activation of Rab1 via GDP-to-GTP-exchange using its GEF domain. This displaces Rab1 from GDI and leads to membrane binding through the C-terminally attached geranylgeranyl-lipids. Subsequently, Rab1:GTP could hypothetically stimulate the AMPylation activity of DrrA by binding to the NC-RBS of the ATase-domain, leading to AMPylation of other recruited Rab1 molecules (see model in Fig. 5f). However, this allosteric activation of DrrA has only been shown in vitro and further in vivo work would be necessary to confirm the relevance of this mechanism for infection by L. pneumophila.

Interestingly, DrrA is present at the LCV only during the early stages of infection, indicating that it may be released from the membrane to the cytosol as maturation of the compartment progresses $^{28}$. Consequently, there may be a risk of global 


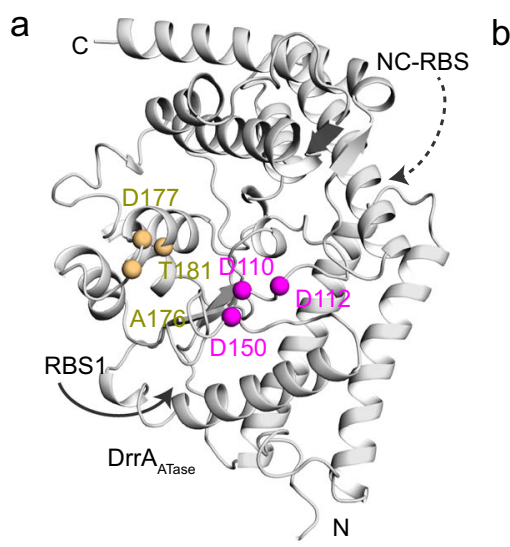

d

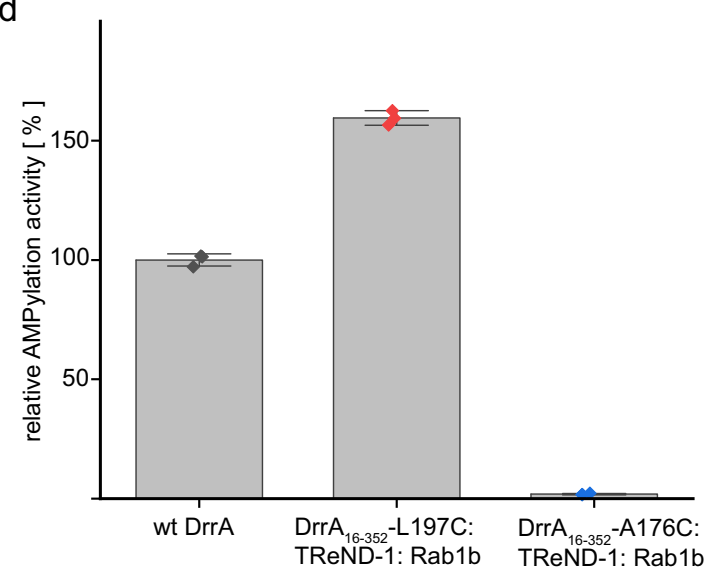

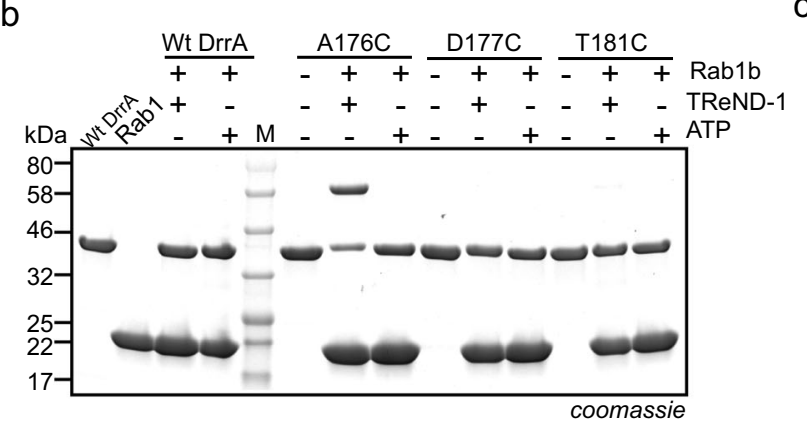

C $\operatorname{DrrA}_{16-352}-\mathrm{A} 176 \mathrm{C}:$ TReND-1:Rab1b

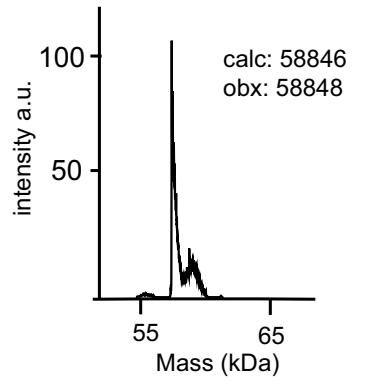

e

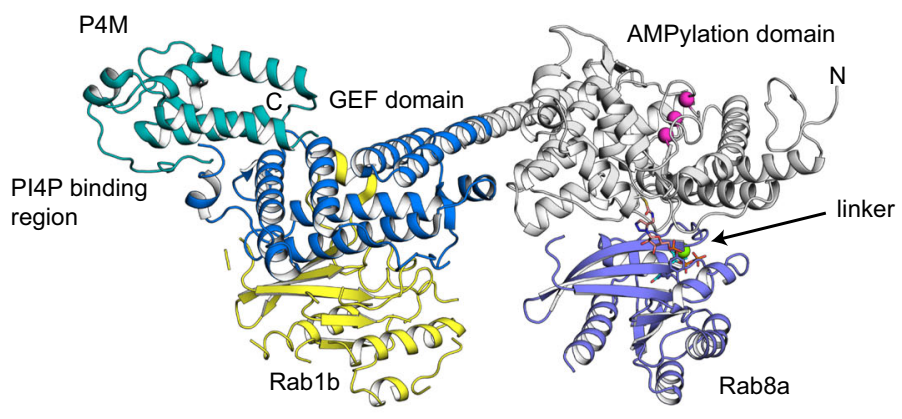

f

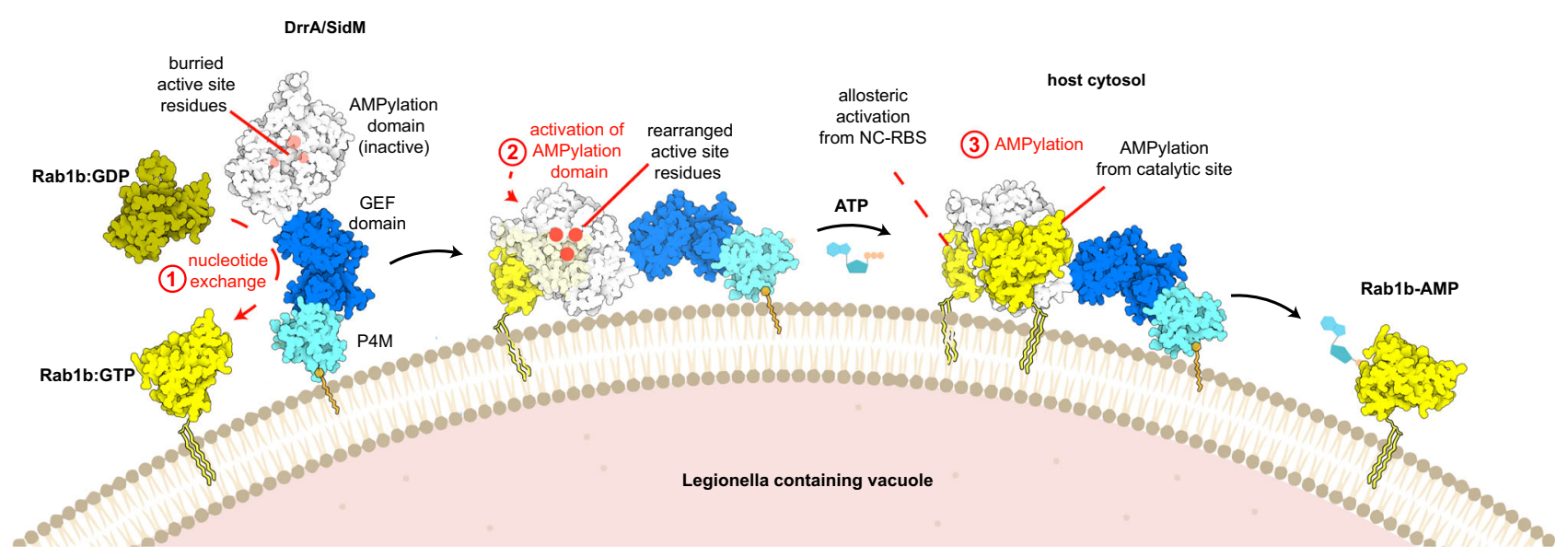

Fig. 5 Formation of the DrrA:Rab complex in RBS1. a Cys substitution sites (A176, D177 and T181, light orange) in DrrA $A_{A T a s e}$. Pink spheres represent the catalytic centre of DrrA $A_{A T a s e}$ RBS1 indicates the conventional site, which contains the catalytic centre. NC-RBS represents the non-canonical site, which is located at the back face of the catalytic centre. b SDS-PAGE shift analysis of DrrA:TReND-1:Rab1b complex formation in the catalytic side (RBS1). $\mathbf{c}$ Intact mass spectrometry analysis of the DrrA ${ }_{16-352}-\mathrm{A} 176 \mathrm{C}$ :TReND-1:Rab1 complex (a.u.: arbitrary units). d AMPylation activity comparison of wt DrrA $\mathrm{A}_{16-352}$ and DrrA:TReND-1:Rab1 complexes using time-resolved tryptophan fluorescence. Data are means \pm SEM from three independent experiments. The $k_{\text {cat }} / K_{M}$ value of wt DrrA is $8.0 \times 10^{5} \mathrm{M}^{-1} \mathrm{~s}^{-1}\left( \pm 2.0 \times 10^{4} \mathrm{M}^{-1} \mathrm{~s}^{-1}\right)$. e Composite model of DrrA produced from the following crystal structures PDB: 6 YX5, 3LOI, 3N6O. The different binding sites of Rab molecules are indicated at the GEF domain and the NC-RBS. The catalytic site of the AMPylation domain (composed by D110, D112, D150) is indicated by magenta spheres. GEF Guanine nucleotide exchange factor; P4M phosphatidylinositol-4-phosphate (PI4P) binding of SidM/DrrA. $\mathbf{f}$ Model of DrrA action at the membrane. The P4M localises DrrA to the membrane by binding to PI4P. The GEF-domain activates Rab1 by GDP-to-GTP exchange, thereby displacing it from GDI and creating membrane-bound Rab1:GTP (yellow ribbons indicate geranylgeranyl lipids). Binding of Rab1:GTP to the NC-RBS of DrrA putatively results in structural rearrangement of the catalytic site, which stimulates the subsequent AMPylation of another Rab1:GTP-molecule. Source data are provided as a Source Data file. 
AMPylation by DrrA with deleterious cellular side effects that may impair infection progression. In this regard, the binding of Rablb:GTP to a regulatory site in the enzyme perhaps functions as a safety mechanism that limits rogue DrrA activity. Since activated Rab GTPases are exclusively membrane-localised, cytosolic DrrA may not be activated by the cytosolic pool of GDP-bound Rab1b ${ }^{29}$. However, in the GDP form, Rab-proteins are complexed with GDI in the cytosol, thereby making switch II inaccessible for AMPylation by DrrA. But the Rab1:GDI complex is weakly dynamic because cellular GEFs are known to contact switch II, leading to GDI displacement by loading the Rab with GTP. Thus, it is conceivable that DrrA could also AMPylate GDP-bound Rab whenever it has been spontaneously dissociated from GDI, explaining the need for an additional safety mechanism, i.e. allosteric activity control of DrrA-AMPylation activity by Rablb:GTP-binding. Nevertheless, the hypothesis remains speculative at this point.

There are several instances where Legionella effectors are activated by host proteins. Legionella effector SidJ requires the host protein calmodulin for activation ${ }^{30,31}$. Upon calmodulin binding, SidJ is activated and polyglutamylates SidE (another Legionalla effector) to inhibit the ubiquitin ligase activity of members of the SidE family. This inactivation is required for successful Legionella replication ${ }^{30,31}$. Secondly, Legionella effector VipD lipase requires Rab5:GTP-dependent activation for phospholipase A1 activity-based targeting of enzymes to endosomes, and this eventually inhibits phagosome maturation to ensure the survival of bacteria within the host ${ }^{32}$. Furthermore, there are multiple examples of effector enzymes from other Gram-negative pathogens such as Salmonella, Yersinia and Vibrio that require host protein-dependent activation ${ }^{33}$. After activation by host proteins, these pathogenic effectors cause numerous PTMs such as ADP-ribosylation ${ }^{34,35}$, phosphorylation ${ }^{36,37}$, acetylation ${ }^{38}$ and proteolytic cleavage ${ }^{39,40}$. However, AMPylation that is dependent on activation by a host protein has not been reported previously. Therefore, DrrA comprises a unique example that requires activation by its substrate.

The mode of regulation of the AMPylation activity of DrrA is different from other DNA polymerase $\beta$-like enzymes and AMP transferases. For example, AMP-transferring GS-ATase is controlled via binding to the regulatory protein PII, and this molecule binds to a linker region connecting the AMP transferase and AMP removase domains of GS-ATase ${ }^{18}$. Complex formation with PII switches on the AMP transferase site of the enzyme, while turning off the hydrolysis activity of the AMP removase ${ }^{41,42}$. The biochemical and structural modes of regulation are clearly different from those of DrrA as GS-ATase is not dependent on the activity of its substrate glutamine synthetase, and does not contain an allosteric site structurally homologous to DrrA. Other AMP transferases are structurally different from DrrA, and are therefore expected to employ different mechanisms of activity regulation. For example, Fic family enzymes, which are known to AMPylate diverse targets, generally harbour an inhibitory helix that safeguards their activity ${ }^{43}$. However, controlling the positioning or displacement of this inhibitory motif has not yet been demonstrated. Furthermore, in some instances, pseudokinases can possess AMP transferase activity, as exemplified by the highly conserved enzyme SelO ${ }^{44}$. This enzyme is controlled by intramolecular disulfide bond formation, but no regulatory binding partner has yet been identified. Thus, the regulation of DrrA by its substrate is unique among AMP transferases.

In summary, we used complementary approaches for the production of low-affinity $\operatorname{DrrA}_{\mathrm{ATase}}$ :Rab complexes using covalent stabilisation by proximity-enabled reactions. Structural investigation and biochemical characterisation revealed a previously unrecognised regulatory site in $\operatorname{DrrA}_{\mathrm{A} \text { Tase }}$ that stimulates
AMP transfer via allosteric binding of the protein substrate (i.e. Rablb:GTP). We speculate that the stimulation of $\operatorname{DrrA}_{\mathrm{ATase}}$ by Rablb:GTP confines AMPylation to the site of DrrA localisation and Rab activation (i.e. the LCV), thereby limiting the potential cytotoxicity of the Legionella enzyme. It will be interesting to see whether this mechanism can also be demonstrated in vivo during infection by $L$. pneumophila.

\section{Methods}

Plasmids and reagents. Full-length DrrA was amplified from L. pneumophila genomic DNA in previous work ${ }^{13}$. For E. coli expression, DrrA truncation constructs were cloned into a modified pSF vector (a gift from Stefanie Pöggeler lab, Georg-August-University Göttingen) via Gibson assembly using Gibson Assembly Master Mix (New England Biolabs, Frankfurt, Germany). Constructs for Rab1 and $R a b 8 a$ were reported previously ${ }^{13,45}$. All point mutations were performed with a Q5 Site-Directed Mutagenesis Kit (New England Biolabs). All plasmids were confirmed by DNA sequencing. Plasmids for genetic code expansion experiments were designed and cloned as described in the Supplementary Information. The synthesis of BrC6K has been reported in detail in Cigler et al. ${ }^{19}$. GppNHp and GDP were purchased from Jena Bioscience, Jena, Germany. The synthesis of TReNDs has been reported previously ${ }^{24}$. Tobacco etch virus (TEV) protease was produced in-house. Lipofectamine LTX for transient transfection in H1299 cells was purchased from Thermo Fisher Scientific (Darmstadt, Germany). MTS agent was purchased from Promega, Walldorf, Germany. Unless otherwise stated, all other reagents were purchased from Sigma (Taufkirchen, Germany) and Carl Roth (Karlsruhe, Germany).

Molecular biology. Cloning of constructs for GCE-experiments in bacterial cells: The pBAD-Rab1b_Q67A-His6 construct was obtained by restriction cloning using HindIII-HF \& NdeI (New England Biolabs) and the templates pBad-His6-TEVEBFP2 and pMal-Rab1b 3-174 opti Q67A and subsequent insertion of the Cterminally His6-Tag via site-directed ligase independent mutagenesis (SLIM) as described by Chiu et al. $^{46}$ using the primer pairs P1 \& P2 and P3 \& P4.

pBAD-RSF1031K-StrepII-DrrA ${ }_{1-647}$ was cloned with NEBuilder ${ }^{\oplus}$ HiFi DNA Assembly (New England Biolabs). The $\operatorname{DrrA}_{1-647}$ gene was amplified using P15 \& P16 (template: pET19mod-DrrA(N451A;R453A;D480A;S483A)). The backbone fragment was derived by PCR with P13 \& P17 (template: pBAD-RSF1031K-StrepII$\left.\operatorname{Drr}_{1-339}\right)$.

pBAD-RSF1031K-StrepII-TEV-DrrA ${ }_{16-352}$ was achieved by NEBuilder ${ }^{\varpi} \mathrm{HiFi}$ DNA Assembly (New England Biolabs). DNA fragments were amplified by PCR using pBAD-RSF1031K-StrepII-DrrA $A_{1-647}$ as template and the primer pairs P18 \& $\mathrm{P} 19$ and P20 \& P21. The assembly yielded the plasmid pBAD-RSF1031K-DrrA 352. In a second step the StrepII-Tag and a TEV-site were inserted N-terminally of $\operatorname{DrrA}_{16-352}$ by Q5 site-directed mutagenesis (New England Biolabs) with the primer pair P22 \& P23.

The pBAD_Duet-Rab1b-Q67A-His6_StrepII-DrrA ${ }_{16-352}$ and pBAD_DuetRab1b-Q67A-His6_StrepII-DrrA $A_{1-647}$ plasmids were cloned using the NEBuilder HiFi DNA Assembly (New England Biolabs). The DNA fragments for the assembly reaction were derived by Q5-PCR using the primer pair P24 and P25 for the fragment containing Rab1b-Q67A-His6 (template: pBAD-Rab1b_Q67A-His6) and P26 and P27 for the fragments encoding StrepII-TEV-DrrA ${ }_{16-352}$ (template: pBAD RSF1031K-StrepII-TEV-DrrA ${ }_{16-352}$ ) or StrepII-DrrA $A_{1-647}$ (template: pBADRSF1031K-StrepII-DrrA ${ }_{1-647}$ ).

Other pBAD-vector or pBAD-Duet-vector variants containing different Rablb and/or DrrA mutants were prepared through substitution using Q5 site-directed mutagenesis (New England Biolabs) on the respective pBAD-vector or pBADDuet-vector template (Supplementary Table 3).

Cloning of constructs for GCE experiments in HEK293T cells: The p(U6PylTU25C)4/EF1a-BrCnKRS plasmid was derived as described by Cigler et al. in $2017^{19}$. The $\mathrm{p}\left(\mathrm{U} 6-\mathrm{PylT}_{\mathrm{U} 25 \mathrm{C}}\right) 4 / \mathrm{EF} 1 \alpha-R a b 1 b-Q 67 A-R 69 T A G-H i s 6-4 x P y l T$ was obtained through restriction cloning. The Rab1b-Q67A-R69TAG_His6 inserts were derived by Q5-PCR with primer M1 \& M2 and inserted into p(U6-PylT $\left.{ }_{\mathrm{U} 25 \mathrm{C}}\right) 4$ / EF1a using NheI-HF and BamHI-HF restriction sites. The pAC-GFP-DrrA 8 -533R70A-Q71A-K74A-D82C plasmid was obtained by Q5 site-directed mutagenesis (New England Biolabs) on pAC-GFP-DrrA ${ }_{8-533}-R 70 A-Q 71 A-K 74 A$. The pAC-GFP$\operatorname{Drr}_{8-533}-D 110 A-D 112 A-D 82 A$ and pAC-GFP-DrrA ${ }_{8-533}-D 110 A-D 112 A \_D 82 C$ plasmids were obtained by Q5 site-directed mutagenesis (New England Biolabs) on pAC-GFP-DrrA $8-533-D 110 A-D 112 A$. For pAC-GFP-DrrA $A_{8-533}-R 70 A-Q 71 A-K 74 A-$ $D 82 C$, the primer pair M5 \& M6 was used. Detailed information for cloning plasmid pAC-GFP-DrrA $A_{8-533}-D 110 A-D 112 A$ will be described in the section of Cloning of constructs for MTS-experiments in H1299 cells. For plasmid pAC-GFP $\operatorname{DrrA}_{8-533}-D 110 A-D 112 A-D 82 A$ and plasmid pAC-GFP-DrrA $8-533-D 110 A-D 112 A-$ $D 82 C$, the primer pairs M7 \& M8 and M9 \& M10 were used, respectively (Supplementary Table 4).

Cloning of DrrA constructs in DrrA:TReND:Rab complexes study with bacterial cells. GFP-TEV-DrrA ${ }_{16-352}$ (wt DrrA) was generated by using GFP-StrepII-Tag- TEVsite- $\operatorname{DrrA}_{16-352}$ with the primer pair wt-F \& wt-R. With this GFP-TEV-DrrA 16-352 
as the template, all the other plasmids are generated by Q5 Site-directed mutagenesis with corresponding primer pairs (New England Biolabs). Rab protein plasmids were cloned before (Supplementary Table 5) ${ }^{13,47}$.

Cloning of DrrA constructs for MTS experiments in H1299 cells. Plasmid pAC-GFP$\mathrm{DrrA}_{16-352}$ was first prepared with Gibson assembly (New England Biolabs). The primer pair $\mathrm{H} 1 \& \mathrm{H} 2$ was used for amplifying the empty pAC plasmid. The DrrA $_{16-352}$ insert was amplified with primer pair $\mathrm{H} 3$ \& $\mathrm{H} 4$. The pAC-GFP-DrrA ${ }_{8-533}$ was produced with the same method. Primer pair H5 \& H6 was used for obtaining the $\operatorname{DrrA}_{8-533}$ insert fragment.

By using pAC-GFP-DrrA $\mathrm{A}_{16-352}$ as a template, pAC-GFP-DrrA $\mathrm{A}_{16-352}$-D110AD112A was obtained with direct transformation of PCR product (primer pairs $\mathrm{H} 7$ \& H8). pAC-GFP-DrrA ${ }_{16-352}$-R70A-Q71A-K74A was obtained with the primer pairs H9 \& H10 by Q5 Site-directed mutagenesis (New England Biolabs).

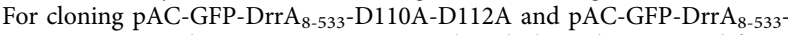
R70A-Q71A-K74A, the pAC-GFP-DrrA $16-352$-based plasmids were used for generating corresponding fragments with overhangs to introduce the mutations (primer pairs $\mathrm{H} 5 \& \mathrm{H} 11$ ). Then the empty pAC backbone was amplified for these fragments (primer pairs H12 \& H13) (Supplementary Table 6).

Protein expression and purification. In general, DrrA constructs are fused to an $\mathrm{N}$-terminal eGFP, which bears an additional $10 \times$ histidine tag. In order to further purify DrrA constructs, a TEV cleavage site was introduced for removing the Nterminal eGFP. Also, to facilitate small-scale purification, another Twin Strep tag was inserted between the TEV site and DrrA (His-GFP-TS-TEV-DrrA). Nevertheless, DrrA proteins were expressed using the E. coli Rosetta2 (DE3) strain under standard conditions. Briefly, when the $\mathrm{OD}_{600}$ of the culture reached 0.6 to 0.8 , $1 \mathrm{mM}$ isopropyl $\beta$ - d-1-thiogalactopyranoside (IPTG) was added to induce protein expression and the temperature was switched from 37 to $20^{\circ} \mathrm{C}$ and maintained overnight for expression in Lysogeny broth (LB) medium. DrrA proteins containing Twin Strep tags were purified with Strep-Tactin Magnetic Microbeads (IBA Lifesciences, Goettingen, Germany) by following the manufacturer's protocol. Twin Strep-tag-free DrrA proteins were purified using Ni-NTA resin. TEV protease was then added to cleave the GFP tag, and proteins were further purified by size exclusion chromatography on a 16/600 Superdex $75 \mathrm{pg}$ column pre-equilibrated with $20 \mathrm{mM}$ HEPES, $100 \mathrm{mM} \mathrm{NaCl}$ and $1 \mathrm{mM}$ Tris(2-carboxyethyl)phosphine (TCEP).

Rab8a and Rab1b were prepared as described previously ${ }^{13,48}$. Briefly, Rablb was fused to a N-terminal His ${ }_{6}$-MBP tag, while Rab8a was fused to a N-terminal $\mathrm{His}_{6}$. Rab1b and Rab8a were expressed in E. coli BL21(DE3). When the $\mathrm{OD}_{600}$ of the culture reached 0.6 to $0.8,1 \mathrm{mM}$ IPTG was added to induce protein expression and the temperature was switched from $37^{\circ} \mathrm{C}$ to $20^{\circ} \mathrm{C}$ and maintained overnight for expression in LB medium. Additionally, Rab8a was co-expressed with the chaperone GroEL/S, and the expression of the GroEL/S chaperone was induced by $1 \mathrm{mg} / \mathrm{mL}$ arabinose when $\mathrm{OD}_{600}$ was 0.4 to 0.5 . After cell disruption using a French press, the lysates were cleared by centrifugation $\left(51,428 \mathrm{~g}, 40 \mathrm{~min}, 4^{\circ} \mathrm{C}\right)$, applied to a Ni-NTA resin (buffer A: $50 \mathrm{mM}$ HEPES- $\mathrm{NaOH} \mathrm{pH} \mathrm{8,} 500 \mathrm{mM} \mathrm{NaCl}, 1 \mathrm{mM}$ TCEP, $1 \mathrm{mM} \mathrm{MgCl}, 1 \mu \mathrm{M}$ GDP) and proteins were eluted with an imidazole gradient (buffer B: buffer A supplemented with $500 \mathrm{mM}$ imidazole). During dialysis with buffer A, the $\mathrm{His}_{6}$-tag was removed by TEV digestion. After reverse Ni-NTA chromatography, Rab proteins were further purified by size exclusion chromatography (buffer: $20 \mathrm{mM}$ HEPES-NaOH pH 8, $100 \mathrm{mM} \mathrm{NaCl}, 1 \mathrm{mM}$ TCEP, $1 \mathrm{mM} \mathrm{MgCl}, 1 \mu \mathrm{M} \mathrm{GDP})$. Proteins were at least $90 \%$ pure. After concentrating to $10 \mathrm{mg} / \mathrm{mL}$, proteins were stored at $-80^{\circ} \mathrm{C}$. Nucleotide exchange for Rabl and Rab8a was performed by following previous protocols. Briefly, 5

mM Ethylenediaminetetraacetic acid (EDTA) was added to Rab1/8a (20 mM HEPES-NaOH pH 8, $100 \mathrm{mM} \mathrm{NaCl}, 1 \mathrm{mM}$ TCEP, $1 \mathrm{mM} \mathrm{MgCl}_{2}, 1 \mu \mathrm{M}$ GDP). A 20 -fold excess of GppNHp was then added and incubated at $4{ }^{\circ} \mathrm{C}$ overnight. For Rab8a nucleotide exchange assays, an additional 5\% (v/v) glycerol was also added for stabilisation, and the buffer was exchanged using NAP columns (GE Healthcare, Munich, Bavaria, Germany). Nucleotide binding to Rab was confirmed by HPLC with a C18 reversed-phase liquid chromatography column.

Expression and purification of BrC6K-bearing Rab1b-proteins. Chemically competent $E$. coli NEB 10-beta cells were co-transformed with both $\mathrm{pBAD}$ Rab1b-Q67A69TAG-His6 (which encodes C-terminally His6-tagged Rablb gene with an amber codon at position R69) and pEVOL-BrCnKRS-PylT (which encodes Mm-BrCnKRS and $\left.M m-t R N A_{C U A}\right)$ plasmids. The transformation was directly inoculated into $50 \mathrm{~mL}$ non-AI medium ${ }^{49}$ containing ampicillin $(100 \mu \mathrm{g} / \mathrm{mL})$ and chloramphenicol $(50 \mu \mathrm{g} / \mathrm{mL})$ and incubated overnight at $37^{\circ} \mathrm{C}, 200 \mathrm{rpm}$. The pre-culture was diluted to an $\mathrm{OD}_{600}$ between $0.04-0.07$ in $500 \mathrm{~mL} \mathrm{AI} \mathrm{medium}{ }^{49}$ supplemented with ampicillin $(100 \mu \mathrm{g} / \mathrm{mL})$, chloramphenicol $(50 \mu \mathrm{g} / \mathrm{mL})$ and nicotinamide $(6 \mathrm{mM})$. The cells were cultivated at $37^{\circ} \mathrm{C}, 200 \mathrm{rpm}$ to an $\mathrm{OD}_{600}$ around $0.2-0.3$ before adding BrC6K $(2 \mathrm{mM})$. Cells were expressed for $12 \mathrm{~h}$ at $37^{\circ} \mathrm{C}, 200 \mathrm{rpm}$.

The cell pellets were resuspended in $30 \mathrm{~mL}$ His-wash buffer containing $50 \mathrm{mM}$ HEPES pH 8.0, $500 \mathrm{mM} \mathrm{LiCl}, 20 \mathrm{mM}$ imidazole, $2 \mathrm{mM} \beta$-mercaptoethanol, $1 \mathrm{mM}$ $\mathrm{MgCl}_{2}$ supplemented with $0.1 \mathrm{mg} / \mathrm{mL}$ DNase I (AppliChem) and two cOmplete ${ }^{\mathrm{TM}}$ Mini EDTA-free protease inhibitor cocktail tablet (Roche). For Rablb proteins the His-wash buffer further contained $0.01 \mathrm{mM} \mathrm{GTP}$. The cell suspension was incubated on ice for $30 \mathrm{~min}$ and sonicated with cooling using an ice-water bath.
The cell lysate was centrifuged $\left(24,000 \times g, 1 \mathrm{~h}, 4^{\circ} \mathrm{C}\right)$ and the cleared lysate was transferred to $1 \mathrm{~mL}$ Ni-NTA slurry (Jena Bioscience) and incubated for $1 \mathrm{~h}$ at $4{ }^{\circ} \mathrm{C}$ slightly shaking. The beads were transferred to a gravity flow column and washed with $200 \mathrm{~mL}$ of His-wash buffer before eluting the proteins in $1 \mathrm{~mL}$ fractions using His-wash buffer supplemented with $500 \mathrm{mM}$ imidazole $\mathrm{pH}$ 8.0. The fractions containing the protein were pooled, concentrated and rebuffered in storage buffer with Amicon Ultra-4 $10 \mathrm{~K}$ NMWL centrifugal filter units (Millipore). Rab1bstorage buffer contained $20 \mathrm{mM}$ HEPES pH 8.0, $150 \mathrm{mM} \mathrm{NaCl}, 5 \mathrm{mM} \mathrm{MgCl}$, $2 \mathrm{mM}$ DTT and $0.01 \mathrm{mM}$ GTP. DrrA-storage buffer contained $20 \mathrm{mM}$ HEPES $\mathrm{pH}$ $8.0,150 \mathrm{mM} \mathrm{NaCl}, 1 \mathrm{mM} \mathrm{MgCl}_{2}$, and $2 \mathrm{mM}$ DTT. Purified proteins were analysed by $15 \%$ SDS-PAGE and/or mass spectrometry and stored at $-80{ }^{\circ} \mathrm{C}$. Protein concentration was determined using NanoPhotometer ${ }^{\circledR}$ N60 (Implen GmbH).

Expression and purification of DrrA variants for GCE-based in vitro experiments. Chemically competent $E$. coli NEB 10-beta cells were transformed with pBADRSF1031K-StrepII-TEV-DrrA $16-352-X X C$ (which encodes N-terminally StrepII-tagged $\operatorname{Drr}_{16-352}$ gene wildtype or cysteine mutants) and directly inoculated into $50 \mathrm{~mL}$ non-AI medium ${ }^{49}$ containing kanamycin $(50 \mu \mathrm{g} / \mathrm{mL})$. Next day the preculture was diluted to an $\mathrm{OD}_{600}$ between $0.04-0.07$ in $500 \mathrm{~mL}$ AI medium ${ }^{49}$ supplemented with kanamycin $(50 \mu \mathrm{g} / \mathrm{mL})$ and incubated for $16 \mathrm{~h}$ at $37^{\circ} \mathrm{C}, 200 \mathrm{rpm}$.

The obtained cell pellets were thoroughly resuspended in $30 \mathrm{~mL}$ of Strep-wash buffer containing $100 \mathrm{mM}$ Tris pH 8.0, $150 \mathrm{mM} \mathrm{NaCl}, 1 \mathrm{mM}$ EDTA and supplemented with $0.1 \mathrm{mg} / \mathrm{mL}$ DNase I (AppliChem) and two cOmplete ${ }^{\mathrm{TM}}$ Mini EDTA-free protease inhibitor cocktail tablets (Roche). The cell suspension was incubated on ice for $30 \mathrm{~min}$ and sonicated with cooling in an ice-water bath. The lysed cells were centrifuged $\left(24,000 \times g, 45 \mathrm{~min}, 4^{\circ} \mathrm{C}\right)$, the cleared lysate added to $500 \mu \mathrm{L}$ of Step-Tactin ${ }^{\circ}$ XT Superflow $50 \%$ Suspension (IBA Lifesciences) and the mixture was incubated with agitation for $5 \mathrm{~min}$ at $4{ }^{\circ} \mathrm{C}$. After incubation, the StepTactin ${ }^{\circ} \mathrm{XT}$ beads were transferred to a gravity flow column and washed with $200 \mathrm{~mL}$ of Strep-wash buffer. The proteins were eluted in $1 \mathrm{~mL}$ fractions with Strep-wash buffer supplemented with $50 \mathrm{mM}$ biotin. The fractions containing the protein were pooled together, concentrated and rebuffered $(20 \mathrm{mM}$ HEPES $\mathrm{pH} 8.0$ $150 \mathrm{mM} \mathrm{NaCl}, 2 \mathrm{mM} \mathrm{DTT}$, and $5 \mathrm{mM} \mathrm{MgCl}_{2}$ ) using Amicon ${ }^{\oplus}$ Ultra- $410 \mathrm{~K}$ NMWL centrifugal filter units (Millipore). Purified proteins were analysed by $15 \%$ SDS-PAGE and stored at $-80^{\circ} \mathrm{C}$. Protein concentration was determined using NanoPhotometer ${ }^{\circ} 60$ (Implen GmbH).

In vitro crosslinking of Rab1b and DrrA using PEPC via GCE. Purified proteins of Rablb variants were mixed with DrrA variants and 1.5-fold excess of Rablb component (Rablb:DrrA = 1.5:1) in Xlink buffer (20 mM HEPES pH 8.0, $150 \mathrm{mM}$ $\mathrm{NaCl}, 2 \mathrm{mM} \beta$-ME, $1 \mathrm{mM} \mathrm{MgCl} 2,0.01 \mathrm{mM}$ GTP) and ATP (final $25 \mu \mathrm{M}$ ) was added. Samples were incubated at $25^{\circ} \mathrm{C}, 200 \mathrm{rpm}$ and samples were taken at different time points ( $30 \mathrm{~min}, 60 \mathrm{~min}, 120 \mathrm{~min}, 240 \mathrm{~min}, \mathrm{O} / \mathrm{N}$ ). Reactions were stopped by directly adding $4 \mathrm{x}$ SDS-Loading buffer and cooking at $95^{\circ} \mathrm{C}$ for $3 \mathrm{~min}$. Samples were analysed by SDS-PAGE.

In vitro crosslinking of Rab1b-R69BrC6K and DrrA-D82C. Non-AMPylated or AMPylated Rab1b variants (Rab1b3-174-Q67A-R69BrC6K-His6 or Rablb3-174Q67A-R69BrC6K-Y77AMP-His6) were mixed with DrrA variants (DrrA16-352D82C or DrrA16-352-D82C-D110A-D112A) in a 1.5:1 ratio (Rab1b:DrrA = 1.5:1) in Xlink buffer (20 mM HEPES pH 8.0, $150 \mathrm{mM} \mathrm{NaCl}, 2 \mathrm{mM} \beta$-ME, $1 \mathrm{mM} \mathrm{MgCl}_{2}$ $0.01 \mathrm{mM} \mathrm{GTP}$ ) and ATP (final $25 \mu \mathrm{M}$ ) was added. Samples were incubated at $25^{\circ} \mathrm{C}, 200 \mathrm{rpm}$ and samples were taken at different time points $(30 \mathrm{~min}, 60 \mathrm{~min}$, $120 \mathrm{~min}, 240 \mathrm{~min}$, o/N or $0 \mathrm{~min}, 30 \mathrm{~min}, 60 \mathrm{~min}, 150 \mathrm{~min}, 300 \mathrm{~min}$ ). Reactions were stopped by directly adding $4 \mathrm{x}$ SDS loading buffer and cooking at $95^{\circ} \mathrm{C}$ for $3 \mathrm{~min}$. Samples were analysed by SDS-PAGE and $\alpha$-His 6 western blot.

In cellulo crosslinking of Rab1b:DrrA using BrC6K. Chemically competent $E$. coli NEB 10-beta cells were co-transformed with either pBAD_Duet-Rab1b-Q67AXXTAG-His6_StrepII-TEV-DrrA $16-352-X X C$ or pBAD_Duet-Rab1b-Q67A-XXTAGHis6_StrepII-DrrA ${ }_{1-647}$ XXC (encoding the C-terminally His6-tagged Rablb gene with an amber codon at different positions and an N-terminally StrepII-tagged $\operatorname{DrrA}_{16-352} / \operatorname{Drr}_{1-647}$ gene with cysteine mutants at different positions) and pEVOL-BrCnKRS-PylT (encoding $M m-B r C n K R S$ and $M m-t R N A_{C U A}$ ) plasmids ${ }^{19}$. Expression was performed under autoinduction (AI) conditions in $10 \mathrm{~mL}$ AI medium ${ }^{49}$. The absorbance of the expression culture at $600 \mathrm{~nm}\left(\mathrm{OD}_{600}\right)$ was determined, $1 \mathrm{~mL}$ of culture was taken, centrifuged $(16,000 \times g, 2 \mathrm{~min}$, room temperature), and the supernatant was discarded. According to the $\mathrm{OD}_{600}$ value, pellets were resuspended in $1 \times$ SDS loading buffer $(100 \mu \mathrm{L}$ of $1 \times$ SDS loading buffer was used for $1.0 \mathrm{OD}_{600}$ units). Samples were heated at $95^{\circ} \mathrm{C}$ for $10 \mathrm{~min}$, centrifuged $(16,000 \times g, 15 \mathrm{~min}$, room temperature), and $10 \mu \mathrm{L}$ was loaded onto a $15 \%$ SDS mini gel. After SDS-PAGE, gels were blotted onto a nitrocellulose membrane using an iBlot 2 Dry Blotting system (Invitrogen, Thermo Fisher Scientific) using programme $\mathrm{P} 0(20 \mathrm{~V}$ for $1 \mathrm{~min}, 23 \mathrm{~V}$ for $4 \mathrm{~min}, 25 \mathrm{~V}$ for $2 \mathrm{~min})$. The membrane was blocked with TBS-T (containing $0.1 \%(\mathrm{v} / \mathrm{v})$ Tween-20) and $5 \%(\mathrm{w} / \mathrm{v})$ skimmed milk powder for $1 \mathrm{~h}$ at room temperature. The blocking solution was removed and either $\alpha$-His6-peroxidase antibody (Roche, Penzberg, Germany) or StrepMABHRP antibody (IBA Lifesciences, Göttingen, Germany) was added at a 1:5000 dilution with TBS-T containing $1 \%(\mathrm{w} / \mathrm{v})$ skimmed milk powder. Incubation was 
performed overnight at $4{ }^{\circ} \mathrm{C}$, the blot was washed three times with TBS-T, and detection was carried out using a WB Imager Fusion Pulse 6 instrument and Amersham ECL Prime western-blotting Detection Reagent (GE Healthcare, Munich, Germany). Uncropped and unprocessed scans of all gels and blots of this manuscript are provided in the accompanying source data file.

For preparative expression of crosslinked complex, expression was performed as described above in $1 \mathrm{~L}$ AI medium ${ }^{49}$. Purification of in vivo crosslinked RablbDrrA complexes involved a two-step procedure comprising $\mathrm{Ni}^{2+}$-affinity and sizeexclusion chromatography. Cell pellets were resuspended in $30 \mathrm{~mL}$ His-wash buffer containing $50 \mathrm{mM}$ 4-(2-hydroxyethyl)-1-piperazineethanesulfonic acid (HEPES) $\mathrm{pH}$ 8.0, $500 \mathrm{mM} \mathrm{LiCl}, 20 \mathrm{mM}$ imidazole, $2 \mathrm{mM} \beta$-mercaptoethanol, and $1 \mathrm{mM}$ $\mathrm{MgCl}_{2}$ supplemented with $0.1 \mathrm{mg} / \mathrm{mL}$ DNase I (AppliChem, Darmstadt, Germany) and two cOmplete Mini EDTA-free protease inhibitor cocktail tablets (Roche). The cell suspension was incubated on ice for $30 \mathrm{~min}$ and sonicated with cooling using an ice-water bath. The cell lysate was centrifuged $\left(24,000 \times g, 1 \mathrm{~h}, 4^{\circ} \mathrm{C}\right)$ and the cleared lysate was mixed with $1 \mathrm{~mL}$ Ni-NTA slurry (Jena Bioscience) and incubated for $1 \mathrm{~h}$ at $4{ }^{\circ} \mathrm{C}$ with gentle shaking. The mixture was poured into a plastic column and washed with $200 \mathrm{~mL}$ of His-wash buffer before eluting bound proteins in $1 \mathrm{~mL}$ fractions using His-wash buffer supplemented with $500 \mathrm{mM}$ imidazole ( $\mathrm{pH}$ 8.0). Fractions containing the protein were pooled and concentrated with an Amicon Ultra-4 $30 \mathrm{~K}$ NMWL centrifugal filter unit (Merck KGaA, Darmstadt, Germany) before applying onto a Superdex 200 Increase 10/300 GL column (GE Healthcare). Separation was performed at a flow rate of $0.5 \mathrm{~mL} / \mathrm{min}$ in SEC buffer $(20 \mathrm{mM}$ HEPES $\mathrm{pH} 8.0,150 \mathrm{mM} \mathrm{NaCl}, 5 \mathrm{mM} \mathrm{MgCl}, 2 \mathrm{mM}$ DTT, $0.01 \mathrm{mM} \mathrm{GTP}$ ), and 0.5 $\mathrm{mL}$ fractions were collected by a 96-well sample collector and analysed using SDSPAGE (15\% mini gels). Fractions containing the complex were pooled, concentrated, and stored at $-80^{\circ} \mathrm{C}$ or used for MS/MS experiments.

LC-MS of purified full-length proteins was carried out on an Agilent 1260 Infinity Series LC system with an Agilent 6210 ESI Single Quadrupole mass spectrometer using a Phenomenex AerisTM Widepore C4 column $(100 \times 2.1 \mathrm{~mm}$, $3.6 \mu \mathrm{m}$ ) (Phenomenex, Torrence, USA). Positive mode was used for the analyzation of protein samples and protein UV absorbance at $280 \mathrm{~nm}$ was monitored. The protein masses were calculated by deconvolution within the MS OpenLab ChemStation software Edition Rev. C.01.07 SR3 [465] (Agilent Technologies). ProtParam webtool was used for calculation of theoretical protein masses, which were manually corrected with masses for unnatural amino acids.

MS/MS acquisition of purified crosslinked protein complexes. Protein samples were lyophilised and resuspended in $200 \mu \mathrm{L}$ buffer X ( $7 \mathrm{M}$ urea, $2 \mathrm{M}$ thiourea) for denaturation. To dissolve proteins completely, samples were sonicated for $10 \mathrm{~min}$ in an ultrasonic bath. Next, $0.2 \mu \mathrm{L}$ of $1 \mathrm{M}$ DTT was added to each sample, mixed, and incubated for $45 \mathrm{~min}$ at room temperature with shaking at $450 \mathrm{rpm}$ to reduce proteins. For alkylation, $2 \mu \mathrm{L}$ of $550 \mathrm{mM}$ iodoacetamide (IAA) was added, mixed, and incubated at room temperature with shaking at $450 \mathrm{rpm}$ for $30 \mathrm{~min}$ in the dark. The reaction was quenched by addition of $0.8 \mu \mathrm{L}$ of $1 \mathrm{M}$ DTT and incubation at room temperature for $30 \mathrm{~min}$ with shaking at $450 \mathrm{rpm}$. A $600 \mu \mathrm{L}$ sample of $50 \mathrm{mM}$ triethylammonium bicarbonate buffer (TEAB) was added so that the $\mathrm{pH}$ was $\sim 8$. Finally, $1.0 \mu \mathrm{L}$ trypsin $\left(0.5 \frac{\mu g}{\mu \mathrm{L}}\right)$ was added and the digest was incubated at $37^{\circ} \mathrm{C}$ overnight with shaking at $450 \mathrm{rpm}$. The reaction was stopped by addition of formic acid (more formic acid was added to ensure the $\mathrm{pH}$ remained 3 or below). For desalting, stage-tipping was performed with a double $\mathrm{C}_{18}$ membrane using Octadecyl C18 $47 \mathrm{~mm}$ Extraction disks (Empore Products, CDS Analytical, Oxford, USA). The membrane was washed three times with $70 \mu \mathrm{L} \mathrm{MeOH,} 70 \mu \mathrm{L}$ buffer E $(80 \%(\mathrm{v} / \mathrm{v})$ acetonitrile, $0.5 \%(\mathrm{v} / \mathrm{v})$ formic acid), and $70 \mu \mathrm{L} 0.5 \%(\mathrm{v} / \mathrm{v})$ formic acid before the digested protein sample was loaded. The membrane was washed three more times with $70 \mu \mathrm{L} 0.5 \%(\mathrm{v} / \mathrm{v})$ formic acid before eluting with twice with $30 \mu \mathrm{L}$ buffer E into a LoBind tube. Samples were lyophilised using a SpeedVac and stored at $-80^{\circ} \mathrm{C}$ until measurement. Before applying samples to the mass spectrometer, they were dissolved in $30 \mu \mathrm{L} 1 \%(\mathrm{v} / \mathrm{v})$ formic acid and filtered using Ultrafree-MCGV centrifugal filters. The filter was washed with $300 \mu \mathrm{L} 1 \%$ (v/v) formic acid by centrifugation at $16,000 \times g$ for $2 \mathrm{~min}$ before the dissolved sample was filtered $(16,000 \times g ; 2 \mathrm{~min})$ and collected into a new LoBind tube.

MS analysis of digested protein samples was performed on an Orbitrap Fusion instrument coupled to an Ultimate 3000 Nano-high-pressure liquid chromatography (HPLC) platform via an electrospray easy source (all Thermo Fisher Scientific). Samples $(7 \mu \mathrm{L})$ were loaded onto a $2 \mathrm{~cm}$ PepMap RSLC C18 trap column $(2 \mu \mathrm{m}$ particles, $100 \mathrm{~A}$, inner diameter $75 \mu \mathrm{m}$; Thermo Fisher Scientific) with $0.1 \%$ trifluoroacetic acid and separated on a $50 \mathrm{~cm}$ PepMap RSLC C18 column ( $2 \mu \mathrm{m}$ particles, $100 \mathrm{~A}$, inner diameter $75 \mu \mathrm{m}$; Thermo Fisher Scientific) at a constant temperature of $50^{\circ} \mathrm{C}$. The gradient was $5-32 \%$ acetonitrile, $0.1 \%$ formic acid $(7 \mathrm{~min} 5 \%, 105 \mathrm{~min}$ to $22 \%, 10 \mathrm{~min}$ to $32 \%, 10 \mathrm{~min}$ to $90 \%, 10 \mathrm{~min}$ wash at $90 \%, 10 \mathrm{~min}$ equilibration at $5 \%)$ at a flow rate of $300 \mathrm{~nL} / \mathrm{min}$. Survey scans $(\mathrm{m} / \mathrm{z}$ $300-1500$ ) were acquired in the Orbitrap with a resolution of 120,000 , and a maximum injection time of $50 \mathrm{~ms}$, with an automatic gain control (AGC) target of 4e5. Most intense ions with charge states of 4-8 and an intensity threshold of 5e3 were selected for fragmentation by high-energy collisional dissociation (HCD) with a collision energy of 30\%. Fragment spectra were again recorded in the Orbitrap with a resolution of 30,000, a maximum injection time of $100 \mathrm{~ms}$, and an AGC target of 5e4. The 'inject ions for all available parallelizable time' option was enabled. Dynamic exclusion was employed with an exclusion duration of $120 \mathrm{~s}$. The overall cycle time was $5 \mathrm{~s}$.

Crosslinking experiments in living mammalian cells. Human embryonic kidney 293 T (HEK293T) cells were cultured in Dulbecco's modified Eagle's medium (Gibco ${ }^{\text {mi }}$ DMEM, Thermo Fisher Scientific) supplemented with 10\% (v/v) FBS (Biochrom) and $1 \%$ Pen-Strep solution $(10 \mathrm{mg} / \mathrm{mL}$ streptomycin, and 10,000 units of penicillin, VWR) at $37^{\circ} \mathrm{C}$ in a humidified chamber with $5 \% \mathrm{CO}_{2}$. One day prior to transfection, cells were seeded ( 3.5 Mio. cells per $100 \mathrm{~mm}$ dish) on Poly-L-lysine coated dishes. Fresh complete DMEM, supplemented with $1 \mathrm{mM} \mathrm{BrC6K}$ was added directly before the transfection of the HEK293T cells using PEI transfection reagent (Sigma-Aldrich) and a 1:3:1 ratio of BrCnKRS-PylT-bearing, Rab1b-PylT-bearing and $e G F P$-DrrA(8-533)-bearing plasmids with a total DNA-amount of $10 \mu \mathrm{g}$ per $100 \mathrm{~mm}$ dish. Cells were cultivated for 24-30 h, harvested and lysed (in lysis buffer $(50 \mathrm{mM}$ Tris- $\mathrm{HCl}$ (pH 8), $150 \mathrm{mM} \mathrm{NaCl}, 20 \mathrm{mM}$ Imidazole; 1x Protease inhibitor solution (VWR)) using freeze-thaw cycles. Cell lysates were cleared by centrifugation $\left(16,000 \times g, 15 \mathrm{~min}, 4^{\circ} \mathrm{C}\right)$ and supernatant was analysed by western-blot analysis using the iBlot" 2 Dry Blotting system (Invitrogen, Thermo Fisher Scientific) and $\alpha$-His6-Peroxidase antibody (1:5000; Roche) or $\alpha$-GFP antibody (1:1000; Santa Cruz Biotechnology; in combination with secondary goat- $\alpha$-mouseHRP (Invitrogen) in a 1:500 dilution). The detection was carried out at WB Imager Fusion Pulse 6 instrument (Vilber Lourmat) using the Amersham ECL Prime western-blotting Detection Reagent (GE Healthcare).

Data analysis and crosslink detection. Raw mass data files were converted to mzML files using MSConvert in ProteoWizard ${ }^{19,50}$. Crosslink searches were then performed with Kojak software version 1.5.5 (http://www.kojak-ms.org) ${ }^{51}$ against a database consisting of Rab1b_Q67A_R69K-DrrA $16-352$, common contaminant proteins downloaded from the Andromeda configuration in the MaxQuant software package ${ }^{52}$, and all reverse sequences. The MS1 and MS2 resolution was set to 120,000 and 30,000 , respectively. Variable modifications included oxidation on methionine $(+15.9949)$ and AMPylation on tyrosine $(+329.0525)$. Static modifications included carbamidomethylation $(+57.02146)$ on cysteine. Three modifications were allowed per peptide. The fragment bin offset was set to 0 and the size was 0.03 . A maximum of three missed cleavages were allowed. The minimum peptide mass was set to $300 \mathrm{Da}$. The precursor mass tolerance was fixed at $10 \mathrm{ppm}$ and the settings for the fragment tolerance were set to fit high-resolution MS2 data (fragment_bin_offset: $0.0 \mathrm{Th}$, fragment_bin_size: $0.03 \mathrm{Th}$ ). The unnatural amino acid was encoded as lysine in our database, hence we searched for crosslinks $(+96.0575)$ between lysines and cysteines, serines, threonines, aspartates, glutamates, and protein N-termini. Additionally, we searched for monolinked species $(+175.9837)$. Annotated spectra were visualised by Kojak Spectrum viewer ${ }^{53}$. Further assessment including hit selection and statistical analysis was performed using custom R scripts (https://github.com/higsch/crosslinkR). Therefore, the Kojak output files *.perc.inter.txt were loaded and each putative crosslink was evaluated whether it contained Rablb and DrrA, and contained no decoy protein at all. Crosslinks fulfilling these criteria are hits (Supplementary Table 1). The script was further used to assess the confidence of the crosslink identifications by setting the hit scores into the context of the score distributions of all decoy and target hits (Supplementary Fig. 2). Please refer to Supplementary Note 1 on the analysis of mass-spectrometric data to get an in-depth explanation of the underlying statistics and our considerations not using an FDR approach here ${ }^{51,53}$

MS proteomics data, Fasta files, and Kojak configuration files have been deposited at the ProteomeXchange Consortium (http://proteomecentral. proteomexchange.org.) via the PRIDE partner repository ${ }^{54}$ under dataset identifier PXD019043.

In vitro AMPylation of Rab1b-Q67A-R69BrC6K-His6. Purified (by Ni-affinity chromatography) Rab1b 3 -174-Q67A-R69BrC6K-His6 was mixed with $\mathrm{DrrA}_{16-352}$ wildtype in a 50:1 Rab1b:DrrA ratio in AMPylation buffer (20 mM HEPES pH 8.0, $150 \mathrm{mM} \mathrm{NaCl}, 1 \mathrm{mM}$ DTT, $1 \mathrm{mM} \mathrm{MgCl}, 0.01 \mathrm{mM}$ GTP) supplemented with an 2.5 excess of ATP compared to the Rab1b component. Samples were incubated at $25^{\circ} \mathrm{C}$, for $3 \mathrm{~h}$. AMPylated Rablb (Rab1b $\left.{ }_{3-174}-\mathrm{Q} 67 \mathrm{~A}-\mathrm{R} 69 \mathrm{BrC6K}-Y 77 \mathrm{AMP}-\mathrm{His} 6\right)$ was purified via size exclusion chromatography using a superdex75 10/300 GL column and SEC buffer (20 mM HEPES pH 8.0, $150 \mathrm{mM} \mathrm{NaCl}, 1 \mathrm{mM}$ DTT, $1 \mathrm{mM}$ $\mathrm{MgCl}_{2}, 0.01 \mathrm{mM}$ GTP).

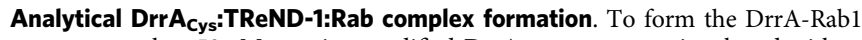
ternary complex, $50 \mu \mathrm{M}$ cysteine-modified DrrA mutants were incubated with $200 \mu \mathrm{M}$ TReND-1, $200 \mu \mathrm{M}$ Rab1:GppNHp (20 mM HEPES-NaOH pH 8, $100 \mathrm{mM}$ $\mathrm{NaCl}, 1 \mathrm{mM}$ TCEP, $1 \mathrm{mM} \mathrm{MgCl}, 1 \mu \mathrm{M}$ GppNHp) overnight at $25^{\circ} \mathrm{C}$. Similar to the formation of the DrrA-TReND-1-Rab1 ternary complex, an additional 5\% (v/v) glycerol was also added to stabilise Rab8a: GppNHp for formation of the DrrA: Rab8a ternary complex. In vitro ternary complex reaction was confirmed by SDSPAGE and MS. The DrrA:TReND-1:Rab complexes were measured using highresolution mass spectra recorded on an Agilent 6230 Series TOF MS instrument, equipped with a Dual AJS ESI ion source and coupled to an Agilent 1290 Infinity II LC system. LC was equipped with an Agilent Poroshell C8 column $(2.1 \mathrm{~mm} \times 75 \mathrm{~mm}$, particle size $5 \mu \mathrm{m}$ ). The flow rate was set to $600 \mu \mathrm{L} / \mathrm{min}$, eluent A consisted of milliQ 
$\mathrm{H} 2 \mathrm{O}+0.1 \%$ formic acid, and eluent $\mathrm{B}$ of acetonitrile $+0.1 \%$ formic acid. Protein samples were injected $(1 \mu \mathrm{L} ; 0.1-0.5 \mathrm{mg} / \mathrm{mL})$ and eluted with linear gradient of $5-60 \%$ $\mathrm{B}$ in $3 \mathrm{~min}$. Mass spectra were recorded in Dual AJS ESI mode with a fragmentor voltage of $250 \mathrm{~V}$ and mass range of $100-1700 \mathrm{~m} / \mathrm{z}, 2$ spectra per second. Acquisition software used was Agilent MassHunter 6200 series TOF/6500 series Q-TOF version B.06.01. Protein mass spectra were analysed with Agilent MassHunter Qualitative Analysis software version B.07.00 with deconvolution in Max Entropy mode (mass steps: 1 Dalton; adduct: proton).

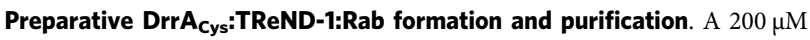
sample of $\mathrm{DrrA}_{16-352}$-L197C, $500 \mu \mathrm{M}$ TReND-1, and $400 \mu \mathrm{M}$ Rab1 or Rab8a (GppNHp) were incubated overnight at $25^{\circ} \mathrm{C}$ or $20^{\circ} \mathrm{C}$. Once the ternary complexes were formed and confirmed by SDS-PAGE, size-exclusion chromatography was performed using a Superdex 26/600 $75 \mathrm{pg}$ column (GE Healthcare) to further purify DrrA:TReND-1: Rab complexes. In order to obtain pure complexes for subsequent experiments (e.g. crystallisation screening), a second run of sizeexclusion chromatography was conducted. For preparing the $\operatorname{DrrA}_{16-352}-\mathrm{A} 176 \mathrm{C}$ : TReND-1:Rab1 complex, a $200 \mu \mathrm{M}$ sample of DrrA $16-352$-A176C, 500 $\mu \mathrm{M}$ TReND1, and $600 \mu \mathrm{M} \mathrm{His}{ }_{10}$-PreScission-Rablb (GppNHp) were incubated overnight at $25^{\circ} \mathrm{C}$ or $20^{\circ} \mathrm{C}$. Following, this complex was purified using Ni-NTA resin. Subsequently, PreScission protease was added to cleave the His tag in Rab1, and then the complexes were further purified by size exclusion chromatography on a 16/600 Superdex 75 pg column (GE Healthcare).

Cytotoxicity analysis of DrrA mutants. In order to evaluate the cytotoxicity of different DrrA mutants, lipofectamine LTX was used for transient transfection of H1299 cells with eGFP, wt DrrA, and various other constructs. At $4 \mathrm{~h}$ after transfection, the culture medium was replaced with $10 \%$ foetal bovine serum (FBS) supplemented Dulbecco's modified Eagle's medium (DMEM). At $24 \mathrm{~h}$ after transfection, GFP-positive cells were sorted in the FACS facility of UKE (using a FACS Aria Fusion, see Supplementary Fig. 16 for gating strategy). Next, 24,000 cells were seeded into three wells of a 96 -well plate. After $24 \mathrm{~h}$, MTS assays were conducted for cytotoxicity analysis. Cell viability was determined by calculating the ratio of the indicated sample relative to the eGFP vector control. Data were analysed using Origin 2019b (Origin software Inc, Northampton, MA 01060, USA) and Graphpad Prism v 5.0 (GraphPad Software, San Diego, CA 92108, USA).

Structure determination of the DrrA-Rab8a complex. The $\operatorname{DrrA}_{16-352}-\mathrm{L} 197 \mathrm{C}$ TReND-1:Rab8a ${ }_{6-176}$ complex in $20 \mathrm{mM}$ HEPES pH 8, $100 \mathrm{mM} \mathrm{NaCl}, 1 \mathrm{mM}$ (TCEP), $1 \mathrm{mM} \mathrm{MgCl}$, and $1 \mu \mathrm{M} \mathrm{GppNHp}$ was concentrated to $14 \mathrm{mg} / \mathrm{ml}$ using a centrifugal filter device (Millipore), and centrifuged to remove debris $(16,000 \times g$, $15 \mathrm{~min}, 4^{\circ} \mathrm{C}$ ) prior to high-throughput crystallisation screening using commercially available screening solutions (NeXtal DWBlock Suites, Qiagen) and robotics (Phoenix, Art Robbins). Complex crystals were obtained by the sitting-drop vapour diffusion method in $0.1 \mathrm{M} \mathrm{BICINE}$ and $2.4 \mathrm{M}$ ammonium sulphate $\mathrm{pH} 9$ at $20^{\circ} \mathrm{C}$. Prior to cryo-cooling and storage in liquid nitrogen, crystals were dipped into reservoir solution supplemented with $2 \mathrm{M}$ lithium sulphate for cryoprotection. Diffraction data were collected at the European Synchrotron Radiation Facility (ESRF, beamline MASSIF-1) and were processed with XDS ${ }^{55}$. Crystals belonged to the P321 space group and the resolution cut-off of $2.15 \AA$ was chosen according to the correlation coefficient of random half datasets (CC $1 / 2)$ at $~ 50 \%{ }^{56-58}$. The structure was solved using the coordinates of the previously reported structures of the N-terminal domain of $\operatorname{DrrA}_{17-210}$ (PDB: 3NKU) and Rab8 (PDB: 4LHV) by molecular replacement in PHASER ${ }^{59}$. Following simulated annealing with PHENIX ${ }^{60,61}$, peaks of density for residues $210-348$ of DrrA16 $16-352$, which were not present in the search model, were clearly visible. The model was completed by iterative cycles of manual model building in COOT $^{62}$ and restraint and TLS (translation/libration/screw) rigid-body motion refinement in REFMAC $5^{63}$. Structure optimisation was carried out using the PDBredo server ${ }^{64}$. Data processing and structure refinement statistics are included in Supplementary Table 1. All structural figures were prepared with PyMol (Schrödinger). Crystallographic data for the DrrA-Rab8a complex have been deposited in the Protein Data Bank (https://www.ebi.ac.uk/pdbe/) under PDB accession code 6YX5.

Temperature-scanning CD measurements. $C D$ signals were recorded on a Chirascan CD Spectrometer (Applied Photophysics, Leatherhead, Surrey, UK) in a $1 \mathrm{~mm}$ cuvette with a bandwidth of $0.5 \mathrm{~nm}$ and a response of $0.5 \mathrm{~s}$. Temperature scanning CD measurements were taken at $222 \mathrm{~nm}$ at a heating rate of $1 \mathrm{~K} / \mathrm{min}$. Data were analysed using Origin $2019 \mathrm{~b}$ and evaluated by the Boltzmann equation to obtain melting temperatures.

AMPylation kinetics of DrrA. The kinetics of Rablb:GppNHp AMPylation by DrrA was monitored via the change in intrinsic Rablb tryptophan fluorescence using an F-2710 fluorescence spectrophotometer (Hitachi, Schaumburg, IL, USA) (excitation wavelength of $297 \mathrm{~nm}$, emission wavelength of $370 \mathrm{~nm}$, excitation slit width of $2.5 \mathrm{~nm}$, an emission slit width of $5 \mathrm{~nm}$ ). The Rablb concentration was $<64 \mu \mathrm{M}$. However, for Rab1:GppNHp concentration $>64 \mu \mathrm{M}$, the emission wavelength was shifted to $390 \mathrm{~nm}$, while keeping other parameters constant. All measurements were conducted in the presence of $100 \mathrm{nM}$ DrrA and 1 unit of pyrophosphate (New England Biolabs) at $25^{\circ} \mathrm{C}$ with GppNHp in buffer $(20 \mathrm{mM}$ HEPES, $100 \mathrm{mM} \mathrm{NaCl}, 1 \mathrm{mM} \mathrm{MgCl} 2,1 \mu \mathrm{M}$ GppNHp, $1 \mathrm{mM}$ TCEP, pH 7.5)

(Supplementary Fig. 17). Data evaluation was performed as previously described ${ }^{14}$ Initial velocities of Rablb-AMPylation were obtained by fitting the AMP-Rablb versus time to equation 1 :

$$
c_{\mathrm{AMP}-\mathrm{Rab} 1}(t)=v * t
$$

where $v$ is the initial velocity, $c_{\mathrm{AMP}-\mathrm{Rab} 1 \mathrm{~b}}(t)$ indicates the concentration of AMPRablb at time $t$ and $t$ is time. For yielding the initial velocity $\left(k_{\mathrm{obs}}\right)$, the initial velocity $v$ was divided by the enzyme concentration.

Hyperbolic curve fitting was performed according to equation 2 :

$$
k_{\text {obs }}=\left(S * k_{\text {cat }}\right) *\left(S+K_{M}\right)^{-1}
$$

where $S$ is the initial concentration of Rab1b, $k_{\text {cat }}$ is the turnover number and $K_{\mathrm{M}}$ is the Michaelis constant.

Sigmoidal curve fitting was performed according to equation 3:

$$
k_{o b s}=k_{c a t} * S^{n} *\left(S^{n}+K_{M}\right)^{-1}
$$

where $n$ is the cooperativity parameter.

To determine the AMPylation rates of different DrrA constructs, timeresolved tryptophan fluorescence was applied. In general, $200 \mu \mathrm{M}$ GTP and $100 \mathrm{nM}$ DrrA $_{\mathrm{GEF}}$ were added for the nucleotide exchange of Rabl $(5 \mu \mathrm{M})$ from GDP to GTP, and AMPylation was initiated by addition of different DrrA constructs $(100 \mathrm{nM})$ (Supplementary Fig. 17). Data evaluation was performed as previously described $^{14}$. In brief, for the determination of catalytic efficiencies $\left(k_{\text {cat }} / K_{\mathrm{M}}\right)$ of AMPylation reactions measured by fluorescence spectrometry, reaction curves were fitted to a single exponential curve according to using equation 4:

$$
F(t)=F_{0}+F_{A} * \exp \left(-k_{o b s} * t\right)
$$

where $F(t)$ is the fluorescence intensity, $F_{0}$ is the minimum fluorescence intensity $F_{\mathrm{A}}$ is the total fluorescence amplitude (i.e., $F_{\max } F_{0}$, with $F_{\max }$ as the maximum fluorescence intensity), and $k_{\mathrm{obs}}$ is the observed rate constant. The observed rate constant $\left(k_{\mathrm{obs}}\right)$ was divided by the applied DrrA concentration $(100 \mathrm{nM})$, yielding $k_{\text {cat }} / K_{\mathrm{M}}$.

Reporting Summary. Further information on research design is available in the Nature Research Reporting Summary linked to this article.

\section{Data availability}

Atomic coordinates and structure factors of the DrrA $\mathrm{A}_{16-352}$ :TReND-1:Rab8 $\mathrm{a}_{6-176}$ complex have been deposited in the Protein Data Bank with accession code 6YX5. The mass spectrometry proteomics data have been deposited at the ProteomeXchange Consortium (http://proteomecentral.proteomexchange.org/) via the PRIDE partner repository, with data set identifier PXD019043. The data that support the findings of this study are available from the corresponding author upon reasonable request. Source data are provided with this paper.

\section{Code availability}

The R scripts used for hit selection and statistical analysis of the Rablb_Q67A_R69K$\operatorname{DrrA}_{16-352}$ crosslinking data are available at https://github.com/higsch/crosslinkR.

Received: 10 July 2020; Accepted: 16 December 2020; Published online: 19 January 2021

\section{References}

1. Isberg, R. R., O'Connor, T. J. \& Heidtman, M. The Legionella pneumophila replication vacuole: making a cosy niche inside host cells. Nat. Rev. Microbiol. 7, 13-24 (2009)

2. Mondino, S. et al. Legionnaires' disease: state of the art knowledge of pathogenesis mechanisms of Legionella. Annu. Rev. Pathol. 15, 439-466 (2020).

3. Cherfils, J. \& Zeghouf, M. Regulation of small GTPases by GEFs, GAPs, and GDIs. Physiol. Rev. 93, 269-309 (2013).

4. Vetter, I. R. \& Wittinghofer, A. The guanine nucleotide-binding switch in three dimensions. Science 294, 1299-1304 (2001).

5. Desnoyers, L., Anant, J. S. \& Seabra, M. C. Geranylgeranylation of Rab proteins. Biochem. Soc. Trans. 24, 699-703 (1996).

6. Tisdale, E. J., Bourne, J. R., Khosravifar, R., Der, C. J. \& Balch, W. E. GTPbinding mutants of Rab1 and Rab2 are potent inhibitors of vesicular transport from the endoplasmic-reticulum to the golgi-complex. J. Cell Biol. 119, 749-761 (1992).

7. Nuoffer, C., Davidson, H. W., Matteson, J., Meinkoth, J. \& Balch, W. E. A GDPbound form of rab1 inhibits protein export from the endoplasmic reticulum and transport between golgi compartments. J. Cell Biol. 125, 225-237 (1994). 
8. Murata, T. et al. The Legionella pneumophila effector protein DrrA is a Rab1 guanine nucleotide-exchange factor. Nat. Cell Biol. 8, 971-977 (2006).

9. Machner, M. P. \& Isberg, R. R. Targeting of host Rab GTPase function by the intravacuolar pathogen Legionella pneumophila. Dev. Cell 11, 47-56 (2006).

10. Brombacher, E. et al. Rabl guanine nucleotide exchange factor SidM is a major phosphatidylinositol 4-phosphate-binding effector protein of Legionella pneumophila. J. Biol. Chem. 284, 4846-4856 (2009).

11. Schoebel, S., Blankenfeldt, W., Goody, R. S. \& Itzen, A. High-affinity binding of phosphatidylinositol 4-phosphate by Legionella pneumophila DrrA. EMBO Rep. 11, 598-604 (2010).

12. Schoebel, S., Oesterlin, L. K., Blankenfeldt, W., Goody, R. S. \& Itzen, A. RabGDI displacement by DrrA from Legionella is a consequence of its guanine nucleotide exchange activity. Mol. Cell 36, 1060-1072 (2009).

13. Muller, M. P. et al. The Legionella effector protein DrrA AMPylates the membrane traffic regulator Rablb. Science 329, 946-949 (2010).

14. Muller, M. P. et al. Characterization of enzymes from Legionella pneumophila involved in reversible adenylylation of Rab1 Protein. J. Biol. Chem. 287, 35036-35046 (2012).

15. Luitz, M. P., Bomblies, R., Ramcke, E., Itzen, A. \& Zacharias, M. Adenylylation of Tyr77 stabilizes Rablb GTPase in an active state: a molecular dynamics simulation analysis. Sci. Rep. 6, 19896 (2016).

16. Hardiman, C. A. \& Roy, C. R. AMPylation is critical for Rab1 localization to vacuoles containing Legionella pneumophila. MBio. 5, e01035-13 (2014).

17. Zhu, Y. et al. Structural mechanism of host Rabl activation by the bifunctional Legionella type IV effector SidM/DrrA. Proc. Natl Acad. Sci. USA 107, 4699-4704 (2010).

18. Xu, Y., Carr, P. D., Vasudevan, S. G. \& Ollis, D. L. Structure of the adenylylation domain of $E$. coli glutamine synthetase adenylyl transferase: evidence for gene duplication and evolution of a new active site. J. Mol. Biol. 396, 773-784 (2010).

19. Cigler, M. et al. Proximity-triggered covalent stabilization of low-affinity protein complexes in vitro and in vivo. Angew. Chem. Int. Ed. 56, 15737-15741 (2017).

20. Nguyen, T. A., Cigler, M. \& Lang, K. Expanding the genetic code to study protein-protein Interactions. Angew. Chem. Int. Ed. 57, 14350-14361 (2018).

21. Chen, X. H. et al. Genetically encoding an electrophilic amino acid for protein stapling and covalent binding to native receptors. ACS Chem. Biol. 9, 1956-1961 (2014).

22. Der, C. J., Finkel, T. \& Cooper, G. M. Biological and biochemical-properties of human rash genes mutated at codon-61. Cell 44, 167-176 (1986).

23. Barthelmes, K., Ramcke, E., Kang, H. S., Sattler, M. \& Itzen, A. Conformational control of small GTPases by AMPylation. Proc. Natl Acad. Sci. USA 117, 5772-5781 (2020).

24. Gulen, B. et al. Identification of targets of AMPylating Fic enzymes by cosubstrate-mediated covalent capture. Nat. Chem. 12, 732-739 (2020).

25. Gavriljuk, K. et al. Reaction mechanism of adenylyltransferase DrrA from Legionella pneumophila elucidated by time-resolved Fourier transform infrared spectroscopy. J. Am. Chem. Soc. 136, 9338-9345 (2014).

26. Guo, Z., Hou, X., Goody, R. S. \& Itzen, A. Intermediates in the guanine nucleotide exchange reaction of Rab8 catalyzed by Rabin8/GRAB. J. Biol. Chem. 288, 32466-32474 (2013).

27. Krissinel, E. \& Henrick, K. Inference of macromolecular assemblies from crystalline state. J. Mol. Biol. 372, 774-797 (2007).

28. Ingmundson, A., Delprato, A., Lambright, D. G. \& Roy, C. R. Legionella pneumophila proteins that regulate Rab1 membrane cycling. Nature 450 365-369 (2007).

29. Blümer, J. et al. RabGEFs are a major determinant for specific Rab membrane targeting. J. Cell Biol. 200, 287-300 (2013).

30. Liu, Y. C. \& Luo, Z. Q. The Legionella pneumophila effector SidJ is required for efficient recruitment of endoplasmic reticulum proteins to the bacterial phagosome. Infect. Immun. 75, 592-603 (2007).

31. Gan, N. et al. Regulation of phosphoribosyl ubiquitination by a calmodulindependent glutamylase. Nature 572, 387-391 (2019).

32. Gaspar, A. H. \& Machner, M. P. VipD is a Rab5-activated phospholipase A(1) that protects Legionella pneumophila from endosomal fusion. Proc. Natl Acad. Sci. USA 111, 4560-4565 (2014).

33. Anderson, D. M., Feix, J. B. \& Frank, D. W. Cross kingdom activators of five classes of bacterial effectors. PLOS Pathog. 11, e1004944 (2015).

34. Fu, H., Coburn, J. \& Collier, R. J. The eukaryotic host factor that activates exoenzyme-S of Pseudomonas-Aeruginosa is a member of the 14-3-3 protein family. Proc. Natl Acad. Sci. USA 90, 2320-2324 (1993).

35. Kodama, T. et al. Identification and characterization of VopT, a novel ADPribosyltransferase effector protein secreted via the Vibrio parahaemolyticus type III secretion system 2. Cell. Microbiol. 9, 2598-2609 (2007).

36. Lee, W. L., Grimes, J. M. \& Robinson, R. C. Yersinia effector YopO uses actin as bait to phosphorylate proteins that regulate actin polymerization. Nat. Struct. Mol. Biol. 22, 248-255 (2015).
37. Lopez, V. A. et al. A bacterial effector mimics a host HSP90 client to undermine immunity. Cell 179, 205-218 (2019).

38. Orth, K. et al. Inhibition of the mitogen-activated protein kinase kinase superfamily by a Yersinia effector. Science 285, 1920-1923 (1999).

39. Calder, T. et al. Vibrio type III effector VPA1380 is related to the cysteine protease domain of large bacterial toxins. Plos One 9, el04387 (2014).

40. Coaker, G., Falick, A. \& Staskawicz, B. Activation of a phytopathogenic bacterial effector protein by a eukaryotic cyclophilin. Science 308, 548-550 (2005).

41. Stadtman, E. R. The story of glutamine synthetase regulation. J. Biol. Chem. 276, 44357-44364 (2001)

42. Itzen, A., Blankenfeldt, W. \& Goody, R. S. Adenylylation: renaissance of a forgotten post-translational modification. Trends Biochem. Sci. 36, 221-228 (2011).

43. Engel, P. et al. Adenylylation control by intra- or intermolecular active-site obstruction in Fic proteins. Nature 482, 107-110 (2012)

44. Sreelatha, A. et al. Protein AMPylation by an evolutionarily conserved pseudokinase. Cell 175, 809-821 e19 (2018).

45. Goody, R. S. et al. The versatile Legionella effector protein DrrA. Commun. Integr. Biol. 4, 72-74 (2011).

46. Chiu, J., Tillett, D., Dawes, I. W. \& March, P. E. Site-directed, ligaseindependent mutagenesis (SLIM) for highly efficient mutagenesis of plasmids greater than 8kb. J. Microbiol. Methods 73, 195-198 (2008).

47. Ernst, S. et al. Legionella effector AnkX displaces the switch II region for Rablb phosphocholination. Sci. Adv. 6, eaaz8041 (2020).

48. Schoebel, S., Cichy, A. L., Goody, R. S. \& Itzen, A. Protein LidA from Legionella is a Rab GTPase supereffector. Proc. Natl Acad. Sci. USA 108, 17945-17950 (2011).

49. Hammill, J. T., Miyake-Stoner, S., Hazen, J. L., Jackson, J. C. \& Mehl, R. A. Preparation of site-specifically labeled fluorinated proteins for 19F-NMR structural characterization. Nat. Protoc. 2, 2601-2607 (2007).

50. Chambers, M. C. et al. A cross-platform toolkit for mass spectrometry and proteomics. Nat. Biotechnol. 30, 918-920 (2012).

51. Hoopmann, M. R. et al. Kojak: efficient analysis of chemically cross-linked protein complexes. J. Proteome Res. 14, 2190-2198 (2015).

52. Cox, J. \& Mann, M. MaxQuant enables high peptide identification rates, individualized p.p.b.-range mass accuracies and proteome-wide protein quantification. Nat. Biotechnol. 26, 1367-1372 (2008).

53. Hoopmann, M. R., Mendoza, L., Deutsch, E. W., Shteynberg, D. \& Moritz, R. L. An open data format for visualization and analysis of cross-linked mass spectrometry results. J. Am. Soc. Mass Spectrom. 27, 1728-1734 (2016).

54. Perez-Riverol, Y. et al. The PRIDE database and related tools and resources in 2019: improving support for quantification data. Nucleic Acids Res. 47, D442-D450 (2019).

55. Kabsch, W. Integration, scaling, space-group assignment and post-refinement. Acta Crystallogr. D. 66, 133-144 (2010).

56. Diederichs, K. \& Karplus, P. A. Better models by discarding data? Acta Crystallogr. D. 69, 1215-1222 (2013).

57. Evans, P. Biochemistry. Resolving some old problems in protein crystallography. Science 336, 986-987 (2012).

58. Karplus, P. A. \& Diederichs, K. Linking crystallographic model and data quality. Science 336, 1030-1033 (2012).

59. McCoy, A. J. et al. Phaser crystallographic software. J. Appl. Crystallogr. 40, 658-674 (2007).

60. Adams, P. D. et al. PHENIX: a comprehensive Python-based system for macromolecular structure solution. Acta Crystallogr. D. 66, 213-221 (2010).

61. Afonine, P. V. et al. Towards automated crystallographic structure refinement with phenix.refine. Acta Crystallogr. D. 68, 352-367 (2012).

62. Emsley, P., Lohkamp, B., Scott, W. G. \& Cowtan, K. Features and development of Coot. Acta Crystallogr. D. 66, 486-501 (2010).

63. Murshudov, G. N. et al. REFMAC5 for the refinement of macromolecular crystal structures. Acta Crystallogr. D. 67, 355-367 (2011).

64. Stamper, G. F., Morollo, A. A. \& Ringe, D. Reaction of alanine racemase with 1-aminoethylphosphonic acid forms a stable external aldimine. Biochemistry 37, 10438-10445 (1998)

\section{Acknowledgements}

We are grateful to the Knut and Alice Wallenberg Foundation, Sweden (KAW 2013.0187 to C.H.), and the Swedish Research Council (VR) for generous support. We acknowledge support from the collaborative research centre SFB1035 (German Research Foundation DFG, Sonderforschungsbereich 1035, Projektnummer 201302640, projects B05 to A.I. and B10 to K.L.). The authors thank the ESRF and SLS for beam time, and the staff of beamlines MASSIF-1 (ESRF) and PX I (SLS) for assistance with crystal testing and data collection. K.L. is a Professor at TUM-IAS and as such acknowledges funding by the Excellence Initiative and the EU Marie Curie COFUND Program. We would like to thank Johannes Buchner, Matthias Feige and Carolin Rulofs at TU Munich. We are thankful to Stephan Sieber for providing MS infrastructure at TU Munich. We are 
thankful for great assistance from other IBS members in UKE, especially Sabine Windhorst, Stefanie Muhs, Gesa König and Vivian Pogenberg. We thank Reinhard Schneppenheim for offering us the H1299 cell line. J.D. was funded by a Chinese Scholarship Council fellowship (2016-2020) for a PhD in TU Munich. B.G. was funded by an Alexander von Humboldt Post-doctoral Fellowship (AvH, 2017-2019). M.S. was funded by the German Academic Scholarship Foundation, as well as the Swedish Childhood Cancer Fund (Barncancerfonden).

\section{Author contributions}

J.D. and B.G. developed the strategy for obtaining the Rab8:DrrA complex. J.D. generated and characterised DrrA mutants, performed kinetic analyses, and analysed DrrA cytotoxicity. M.W. designed and conducted genetic code expansion experiments and in cellulo crosslinking. B.G. assisted writing the manuscript. M.S. identified the UAA crosslink site by mass spectrometry. C.P. measured the TReND-1 based complexes by high-resolution mass spectrometry. S.S. solved and refined the Rab8:DrrA crystal structure. J.D., B.G., S.S., and A.I. analysed and interpreted the complex structure. C.H. designed and contributed TReNDs. K.L., C.H. and A.I. designed the concept of this study. K.L., C.H., S.S. and A.I. provided lab infrastructure. J.D., K.L. and A.I. wrote the manuscript; M.W., M.S., B.G. and C.H. edited the manuscript. A.I., S.S. and K.L. provided oversight over the project.

\section{Funding}

Open Access funding enabled and organized by Projekt DEAL.

\section{Competing interests}

The authors declare no competing interests.

\section{Additional information}

Supplementary information is available for this paper at https://doi.org/10.1038/s41467020-20702-2.

Correspondence and requests for materials should be addressed to K.L., S.S. or A.I.

Peer review information Nature Communications thanks Jacqueline Cherfils and the other, anonymous, reviewer(s) for their contribution to the peer review of this work. Peer reviewer reports are available.

Reprints and permission information is available at http://www.nature.com/reprints

Publisher's note Springer Nature remains neutral with regard to jurisdictional claims in published maps and institutional affiliations.

\section{(c) (i)}

Open Access This article is licensed under a Creative Commons Attribution 4.0 International License, which permits use, sharing, adaptation, distribution and reproduction in any medium or format, as long as you give appropriate credit to the original author(s) and the source, provide a link to the Creative Commons license, and indicate if changes were made. The images or other third party material in this article are included in the article's Creative Commons license, unless indicated otherwise in a credit line to the material. If material is not included in the article's Creative Commons license and your intended use is not permitted by statutory regulation or exceeds the permitted use, you will need to obtain permission directly from the copyright holder. To view a copy of this license, visit http://creativecommons.org/ licenses/by/4.0/.

(C) The Author(s) 2021 